\title{
Real-Time Remote-Health Monitoring Systems: a Review on Patients Prioritisation for Multiple-Chronic Diseases, Taxonomy Analysis, Concerns and Solution Procedure
}

\author{
K. I. Mohammed ${ }^{1}$ - A. A. Zaidan ${ }^{1}$ - B. B. Zaidan ${ }^{1}$ - O. S. Albahri ${ }^{1}$ - M. A. Alsalem ${ }^{2}$ - A. S. Albahri ${ }^{3} \cdot$ Ali Hadi $^{4}$ M. Hashim $^{1}$
}

Received: 3 September 2018 / Accepted: 30 May 2019 / Published online: 11 June 2019

(C) Springer Science+Business Media, LLC, part of Springer Nature 2019

\begin{abstract}
Remotely monitoring a patient's condition is a serious issue and must be addressed. Remote health monitoring systems (RHMS) in telemedicine refers to resources, strategies, methods and installations that enable doctors or other medical professionals to work remotely to consult, diagnose and treat patients. The goal of RHMS is to provide timely medical services at remote areas through telecommunication technologies. Through major advancements in technology, particularly in wireless networking, cloud computing and data storage, RHMS is becoming a feasible aspect of modern medicine. RHMS for the prioritisation of patients with multiple chronic diseases (MCDs) plays an important role in sustainably providing high-quality healthcare services. Further investigations are required to highlight the limitations of the prioritisation of patients with MCDs over a telemedicine environment. This study introduces a comprehensive and inclusive review on the prioritisation of patients with MCDs in telemedicine applications. Furthermore, it presents the challenges and open issues regarding patient prioritisation in telemedicine. The findings of this study are as follows: (1) The limitations and problems of existing patients' prioritisation with MCDs are presented and emphasised. (2) Based on the analysis of the academic literature, an accurate solution for remote prioritisation in a large scale of patients with MCDs was not presented. (3) There is an essential need to produce a new multiple-criteria decisionmaking theory to address the current problems in the prioritisation of patients with MCDs.
\end{abstract}

Keywords Healthcare services $\cdot$ Remote health monitoring system $\cdot$ Sensor $\cdot$ Priority $\cdot$ Triage $\cdot$ Chronic disease $\cdot$ Multiple chronic diseases

This article is part of the Topical Collection on Systems-Level Quality Improvement

A. A. Zaidan

aws.alaa@gmail.com; aws.alaa@fskik.upsi.edu.my

K. I. Mohammed

khalid_ib81@yahoo.com

B. B. Zaidan

bilalbahaa@fskik.upsi.edu.my

O. S. Albahri

osamahsh89@gmail.com

M. A. Alsalem

mohammed.asum@gmail.com

A. S. Albahri

ahmed.bahri1978@gmail.com
Ali Hadi

alihadi.mohsin@gmail.com

M. Hashim

mashitoh.hashim@fskik.upsi.edu.my

1 Department of Computing, Universiti Pendidikan Sultan Idris, Tanjong Malim, Perak, Malaysia

2 College of Administration and Economic, University of Mosul, Mosul, Iraq

3 College of Engineering, University of Information Technology and Communications, Baghdad, Iraq

4 Presidency of Ministries, Establishment of Martyrs, Baghdad, Iraq 


\section{Introduction}

Telemedicine has been increasingly used in healthcare because it offers several benefits, such as providing the healthrelated information of patients [1-7] and remote healthcare services [8-14]. Telemedicine is a remote medical practice in which different healthcare providers cooperate and permit their collaborative efforts in the diagnosis or treatment of a disease [15-18]. Remote healthcare services can be beneficial for patients living in isolated communities and far-flung regions, provided that they receive professional care from physicians or specialists who travel to visit them [19-22].

Triage in telemedicine is the concept of sorting patients in order of treatment necessity in a large-scale emergency. In other words, it is the process of assessing and prioritising care for all patients and casualties [23-27]. A prioritisation process is often conducted to ensure that care is given in a suitable and timely manner $[28,29]$. The early identification of critically ill patients and their stratification into priority levels upon acceptance to the emergency department (ED) are necessary for the quality and integrity of emergency medicine [30-34]. Hence, patient prioritisation aims to identify patients who can safely wait and those who cannot [35]. Patient prioritisation improves fairness and decreases urgent patients' waiting times can affect the distinctions among areas because it efficiently appropriates available resources within each region [36, 37]. A patient's case must be the main basis in assessing priority according to medical guidelines [38].

Remote healthcare prioritisation systems have gained considerable attention because of their significant role in the lives of people [39, 40]. Remote prioritisation means triaging patients and identifying their priority for treatment and transportation to hospitals after the evaluation of their vital signs [41]. For remote patients, continuous monitoring from a distant hospital is highly desirable because it ensures adequate care and provides suitable guidelines for proper medication [42]. Moreover, remote patient care is currently becoming a major concern in healthcare services [43]. Patients with multiple chronic conditions (e.g., chronic heart disease [CHD], chronic blood pressure [BP] disease, fall detection and diabetes) are at great risk of experiencing poor daily functioning [26]. Multiple chronic diseases (MCDs) contribute to frailty and disability. Functional limitations often complicate access to healthcare, interfere with self-management and necessitate reliance on caregivers [44]. Remote home patients, especially the elderly with MCDs, are at critical risk of harm in various situations [45]. Thus, prioritisation processes for MCDs are important to the continuous care of remote patients in pervasive environments.

A comprehensive and deeply literature review is essential for highlighting and scrutinising the process of patient prioritisation with MCDs in telemedicine applications. Challenges in patient prioritisation and open issues within telemedicine applications require further investigation (Fig. 1).

The rest of the study is organised as follows: Section 2 systematically reviews existing patients' prioritisation studies. Section 3 presents RHMS for MCDs. Section 4 presents concern for prioritisation process. Section 5 presents multiplecriteria decision-making (MCDM) techniques as a solution for problems in the prioritisation of patients with MCDs. Section 6 summarises the research.

\section{Systematic review protocol for patients' prioritisation}

The scope of this study was established by using the keywords 'telemedicine', 'triage', 'priority' and 'sensor'. Other telemedicine studies, such as surveys and reviews, were excluded. The scope was limited to English literature but considers all healthrelated areas. Three digital databases were searched for target articles: (1) the Science Direct database offering access to science, technology and articles in highly reliable journals; (2) the IEEE Xplore Library of technical literature in engineering and technology and (3) the Web of Science (WoS) service $[46,47]$. These databases index cross-disciplinary research in sciences, social sciences, arts and humanities [48, 49]. The justification behind this selection is to cover scientific and technical literature and provide an extensive insight into researchers' efforts in a wide yet relevant range of disciplines [50-52]. Study selection involves searching literature sources and the subsequent three iterations of screening and filtering [53]. The first iteration excludes duplicate articles and collects only articles from the last six years (2012-2017) by using the Mendeley software. The second iteration filters the reading titles and abstract and excludes articles out of our domain. Lastly, the third iteration filters a full-text reading and excludes articles out of our domain and out of our criteria, as shown in Fig. 2. All iterations apply the same eligibility criteria and followed by screening and reviewing. The search was conducted in Science Direct, IEEE Xplore and WoS databases via their search boxes on January 2017. We used a combination of keywords that contained 'medical system'; 'Telemonitoring'; 'E health'; 'Telemedicine'; 'Telehealth'; 'healthcare services'; 'remote monitoring' and 'mobile physician' in different variations combined by the operators 'OR' and 'AND', followed by 'Triage'; and 'Priority' in different variations combined by the operators 'OR' and 'AND' and by 'sensor'. The exact query text is shown at the top of Fig. 2. We further used the options in each search engine to exclude book chapters and other types of reports other than a journal and conference articles, considering that those two venues are the most probable to include up-to-date and proper scientific works relevant to our survey in this emergent trend of telemedicine. Every article that met the criteria was included. We 
Review and analysis on patients' prioritisation for multiple-chronic diseases

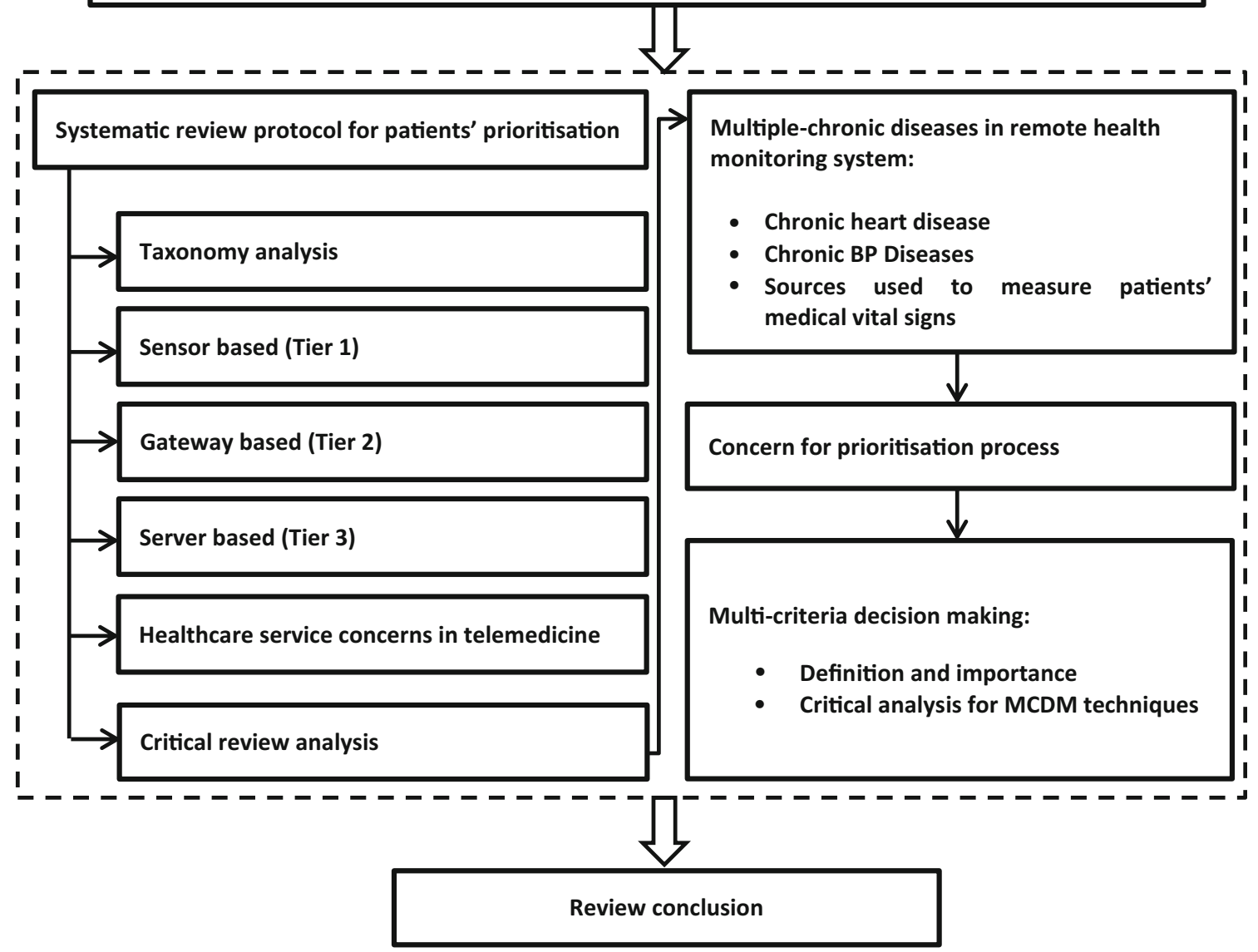

Fig. 1 Review and analysis structure

set an initial target of mapping the space of research in our title into a general and coarse-grained taxonomy composed of two categories. These categories were derived from a presurvey of the literature with no constraint (Google Scholar was used to obtain a preview of the landscape and directions in the literature). After the initial removal of duplicates, articles were excluded in three iterations of screening and filtering if they did not fulfil the eligibility criteria. The exclusion reasons include the following: (1) the article is non-English, (2) the focus is on a specific aspect of healthcare technology and (3) the target is the general telemedicine application. Herein, we focused on healthcare services, patient prioritisation, patient triage, disaster management, network failure, sensors in telemedicine and security of telemedicine.

\section{Taxonomy analysis}

The initial query search obtained 3064 articles (i.e. 911 from Science Direct, 1496 from IEEE Xplore and 657 articles from WoS) published within 2007 and 2017. Unfortunately, we were unable to download only two scientific articles from WoS because of accessibility issues. With three categories, the articles filtered by the following sequence were adopted. Of the 1612 papers collected roughly from the last six years (2012-2017), 62 were duplicates. After scanning the titles and abstracts, we further excluded 1264 papers. Then, 163 papers were excluded at the last full-text reading excluded. A total of 123 papers were finally obtained, which we read thoroughly to determine a general map for the conducted research on this emerging topic. Through taxonomy analysis, specific patterns and general categories were observed in the telemedicine architecture. The mapping and domains of telemedicine technology are illustrated in Fig. 3.

\section{Sensor based (tier 1)}

This category highlights the challenges and sensor techniques which enables the continuous monitoring of healthcare services. Wireless body area networks (WBANs) include small wireless sensors that collect and carry patients' vital sign information [54]. In the literature, sensor-based (Tier 1) telemedicine applications have been discussed in seven domains.

The first domain is ontology, which focuses on the extended principles of topology that deeply explains wearable 
Query

'Medical system' OR 'Tele monitoring' OR 'E health' OR 'Telemedicine' OR 'Telehealth' OR 'healthcare services' OR 'Remote Monitoring' OR ‘mobile doctor' AND 'Triage' OR 'Priority' AND ‘sensor'

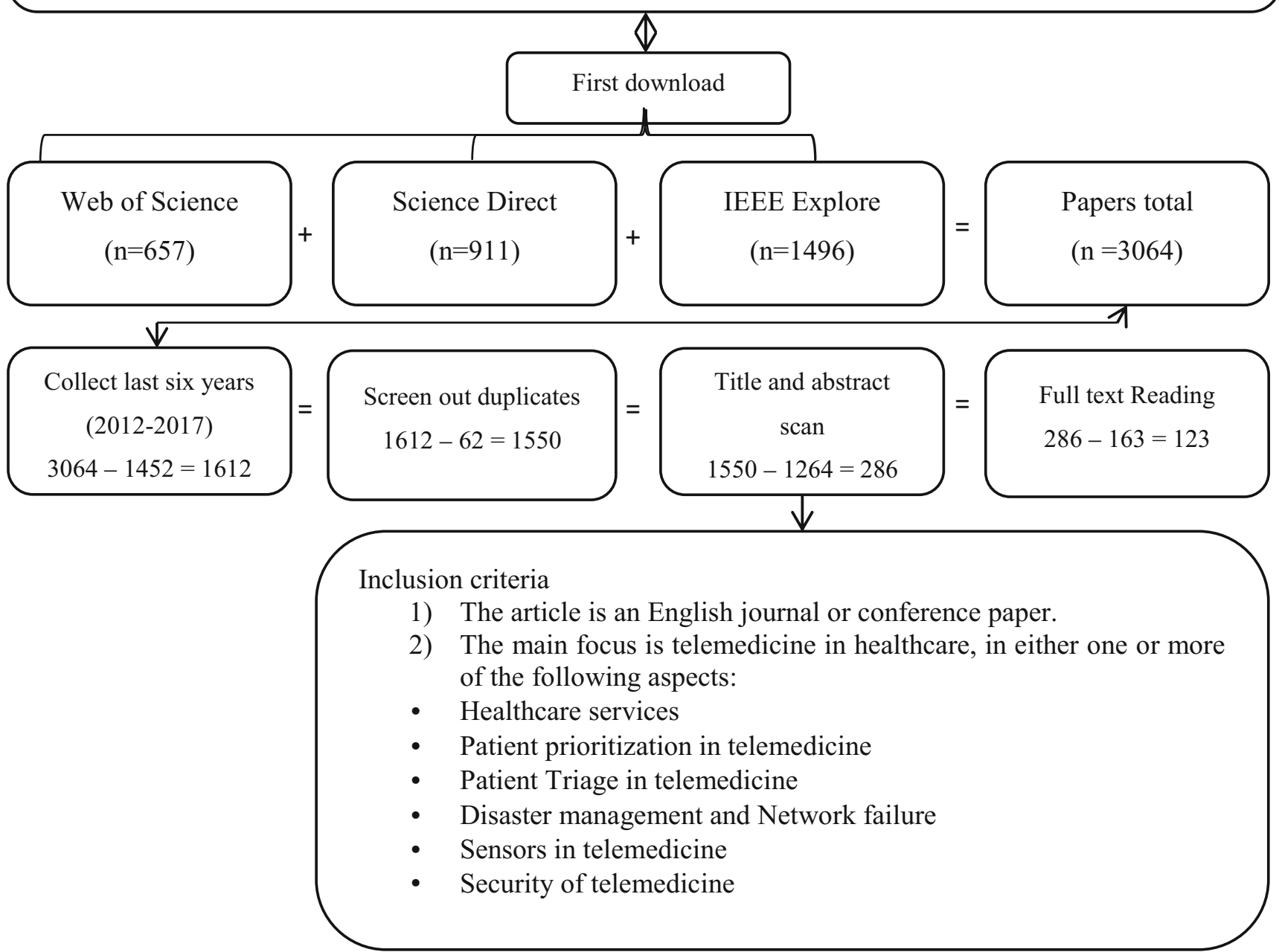

Fig. 2 Study selection flowchart containing the exact query and inclusion criteria

platforms containing 'mainstream magnetic and inertial measurement units' [55].

The second domain is reliability, which contains three areas in the literature. The first area is congestion control; some studies focus on detecting congestion and control protocols for remotely monitoring the health and healthcare conditions of patients by WBANs [56, 57]. The congestion management protocol is the main contribution of other studies and is widely used in medical applications $[58,59]$. The second area is packet delay and link quality. In [60], 'real-time publish-subscribed middleware functions' were established in healthcare applications. In [61], techniques for multiple attribute decision analysis and scheme of decision were examined. Another study presented a healthcare system architecture that addresses dynamic behaviour and various traffic in WBAN [62]. Another study was focused on scheme of health tracking and on sensor structure with simulated results [63]. Another study attempted to enhance the quality of the Telehealth system through its proposed infrastructure [64]. Meanwhile, in the third area, which is electromagnetic interference (EMI), the number of users is reduced through keeping EMI on medical sensors at a satisfactory level [65].

The third domain is energy efficiency. Some studies were focused on 'higher energy efficiency and robust data transmission' by reducing energy consumption and prolonging network lifetime by increasing the predictability of a system [54, 66-69]. The last study introduced green-cloud-assisted healthcare services on WBANs [70].

The fourth domain is the quality of service (QoS). Some studies presented QoS for WBANs and provided parameters for understanding the QoS-performance criterion [54, 66-69]. 


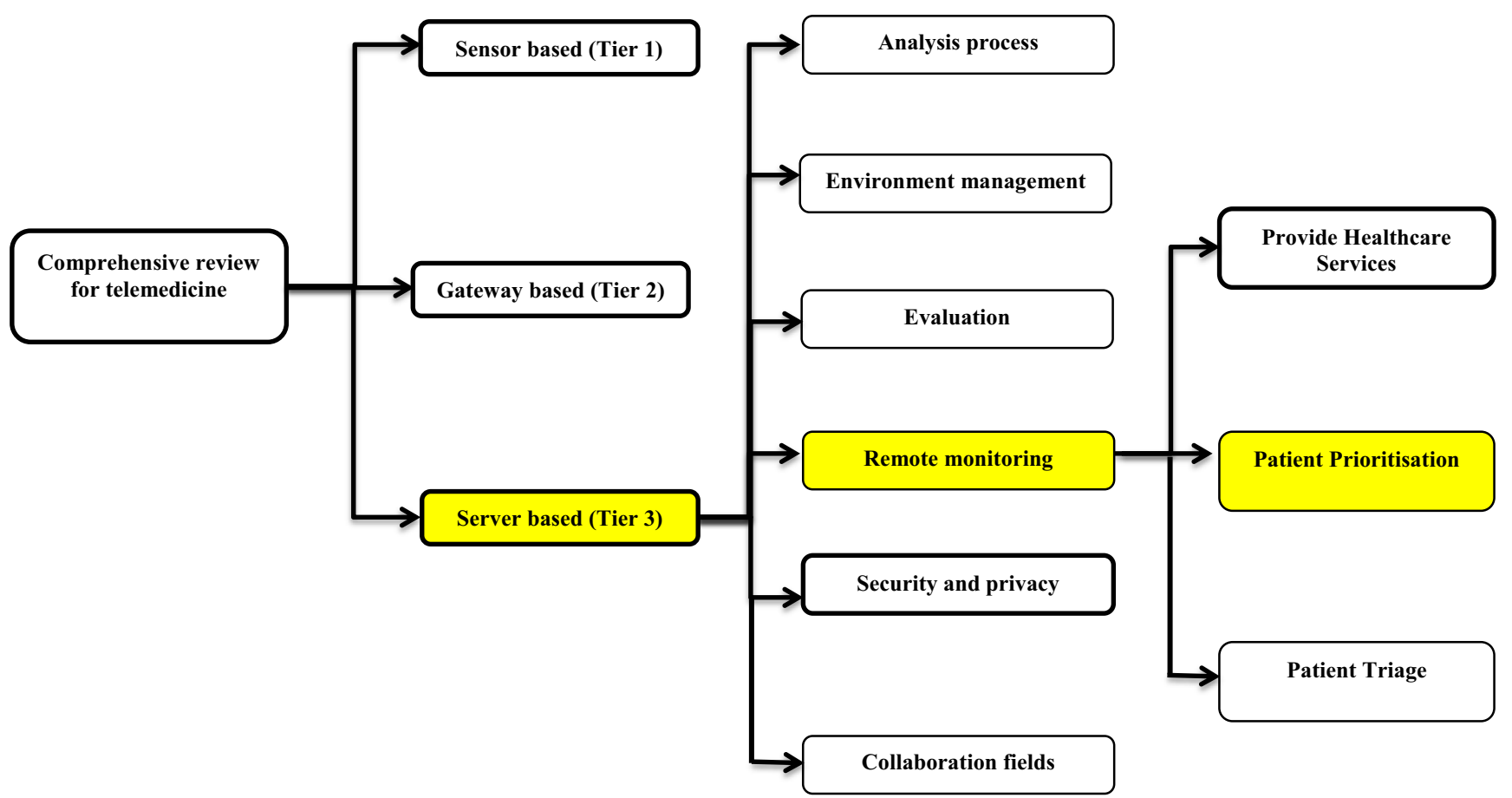

Fig. 3 Research taxonomy on telemedicine applications

The fifth domain is security and privacy. One of the studies focused on securing wireless communication for sensor nodes and implemented a prototype application for biomedical sensors [71]. Another article presented secure logging for information collected from the WSN nodes [72]. In [73], researchers introduced various security levels in BANs for medical devices to prevent long-distance attacks. A cryptographic hash algorithm for preserving data integrity was proposed in [74]. Lastly, a study integrated the healthcare enterprise into various eHealth/mHealth systems [75].

The sixth domain is evaluation and assessment. One of the studies focused on the performance evaluation of the IEEE standard 802.15.6 [76, 77]. Another study evaluated the efficiency of wearable health devices to enhance communication among healthcare units [78]. Meanwhile, a study introduced an evaluation of miniature wireless vital-sign monitor for potential trauma triage in intensive care units [79].

The seventh domain is prioritisation over the sensor. Some studies presented priority-based time-slot allocation scheme for WBANs to achieve energy efficiency [80-82]. Another study proposed healthcare applications based on the integrated 'cross-layer routing protocol' and 'cross-layer medium-access channel protocol' [83]. Another study proposed two new scheduling algorithms to satisfy QoS requirements in WBAN networks and overcome the starvation mode of packets without the highest priority [84]. Another article used 'non-pre-emptive priority queue discipline' to present a new traffic-sensitive WBAN [85]. Another study presented priority based on 'congestion-avoidance hybrid scheme' for WSN to provide an efficient mechanism that reduces power consumption [86]. Furthermore, a study proposed the medium-access control (MAC) protocol for considering the priorities of several biosignals with different characteristics and setting up minimum packet delay time to guarantee data effectiveness [87]. Other studies focused on the improvement of the performance of 'MAC protocol of WBANs' for the prioritisation of numerous vital signs to ensure data efficiency [88, 89]. Finally, [90] presented probabilistic real-time systems in BASNs with fixed-priority pre-emptive scheduling.

\section{Gateway based (tier 2)}

Gateway is a common literature term that refers to using any wireless devices, such as phones and other devices for communication, which are often used for educating consumers regarding the use of 'preventive healthcare services' in medical care [91, 92]. Gateway-based (Tier 2) telemedicine applications have been studied in different domains.

The first domain is a mobile user interface (MUI). In [93], the approach depends on a context and rules to design MUIs for mHealth use.

The second domain is evaluation. In [94], medical alarm dissemination feasibility was explored, and mobile phones were used in the failure or congestion cases of network in an urban environment.

The third domain is treatment support and disease surveillance. The key purpose of both topics is to decrease the harm caused by the outbreak, epidemic, predicted and pandemic cases, then to observe and identify the factors causing such situations. In some articles, patients were allowed to monitor 
and manage chronic diseases. Meanwhile, chronic disease management and patient monitoring on body temperature, heart rate and blood pressure were conducted other studies $[95,96]$. Applications that can be used for some diseases, such as fever, high BP and diabetes, were introduced and used in devices based on biometrics, such as tensiometers, thermometers and glucometers [97, 98]. Other studies presented systems based on social media [99], which presented the tracking of children's vaccination coverage in rural areas. In [100], the researchers developed a software for dengue prevention in Sri Lanka. Patients with major depression were treated by a method presented in [101], in which patients were allowed to obtain daily interactive sessions featuring personalised and adaptive, as well as continuous monitoring of patients with Parkinson's disease [102]. In [103], the researchers presented systems for personal coaching; the systems combines 'onbody sensing with smart reasoning and context-aware feedback'. Lastly, eating-disorder treatment was studied in [104].

The fourth domain is disaster management. The management and organisation of all the responsibilities and resources necessary for addressing emergency cases cover all the humanitarian aspects, that is, recovery, preparedness and response, to decrease the harmful effects of natural disasters, indicating the system of mHealth management. These studies applied the system of mHealth in a 'tsunami-stricken disaster' scenario, as well as proposed a platform for developing 'a field accident and emergency centre intelligent monitoring system' $[105,106]$. The study of [107] presented an assessment system of patients in real time on the basis of 'mobile electronic triaging' and has been performed by sensor-detected information and crowdsourcing. Other systems for rescue operations in urban areas are based on situational awareness, indoor and outdoor [108]. Tracking system of pilgrims in holy spaces was also developed [109]. In [110], a network called 'Medical Body Area Network' was presented, which gathers triage and general physiological data in case of a disaster by using sensors.

The fifth domain includes the integration of data source and aggregation. Integration refers to the incorporation of multiple sources of data that can be heterogeneous for the utilisation of healthcare data in the clinical field, production of a mobile operator and development of solutions that support the connection between patients and healthcare providers. Aggregation is demonstrated in 'Mobile Aggregation Centres' and enhances system scalability and flexibility. The modelling of linear data with an expert priority was demonstrated in [111]. The theory-based approach and the design description among architecture, algorithmic and technology systems were reported in [112]. Healthcare environments in virtual organisations were integrated in [113]. Two architectures for the aggregation of healthcare data. The first architecture, which enables aggregation through cloud-assisted WBAN, was first presented in [114]. In the second architecture, a priority scheme for cloud-assisted WBANs with privacy maintenance, was presented in [115].

The sixth domain is network management, in which any wireless technologies, such as mobile phones, can be used to support the objectives of mobile health through SMS, calling and mobile phone applications, as well as to develop and enhance the management of mobile healthcare network. A capacity-sharing scheme for allocating radio resources depends on a priority-aware pricing was proposed in [116]. In [117], a mechanism of incentive compatibility in e-health networks to schedule the transmission. Scheme supports multiuser sharing proposed in [118]. Another study presents patient monitoring using mobile ad-hoc network to classify challenges to improve the communication reliability [119].

'Ambient Assisted Living (AAL)' is the seventh domain. This domain focuses on patient tracking and monitoring, particularly for patients staying alone at home or solely receiving treatment in hospitals. Models that simulate daily activities of a person living alone at home were proposed in $[120,121]$ and [122] for the implementation of data-driven, cloud-based and semantic software through the knowledge acquired by such models. 'Internet-of-Things (IoT)' and 'Cyber-physical Systems' are more capable of predicting and more clever for home/office in daily life and hospitals, as presented in [123].

The eighth domain is the decision support system, which is a computerised system that assists decision-making processes and provide precise diagnosis for any disease. Decisions related to context awareness sets and processing are enhanced, and thus the presentation of information to professional healthcare are improved [124]. In [125], DSSs based on knowledge embeddable in mobile devices was proposed. Another study focused on discovering and decreasing false alarms produced by the monitoring sensors of patients [126].

\section{Server based (tier 3)}

This category is responsible for the real-time remote monitoring of patients through the use of a remote computer in telemedicine environment. The vital signs of patients are collected and then sent to a telemedicine server for further analysis and investigation. After analysing the vital signs, the medical server can support medical professionals by identifying appropriate healthcare services for patients remotely [23]. In the literature, server-based telemedicine applications have been studied in different domains.

The first domain is the analysis process. Some studies conducted huge data analysis on sensing techniques that can improve the efficiency of healthcare services [127, 128]. Meanwhile, 'activity pattern mining' was analysed in [129].

The second domain is environment management. Some studies focused on managing the data collection process of elderly people within hospitals $[130,131]$. Another study 
focused on helping physicians to improve management and reveal the issue of overcrowding [132].

The third domain is the evaluation and assessment domain. Some studies evaluated the features and effectiveness of common telehealthcare systems [133-135].

The fourth domain is the security and privacy domain. The vital signs collected from medical sensor networks were secured by ensuring confidentiality and integrity [136]. Another study focused on securing e-Healthcare society by proposing various actors to communicate securely [137].

The fifth domain is collaboration field, which is divided into two areas: cooperative environment and tele-expertise between professionals. Cooperative environment involves ubiquitous healthcare in the sharing of medical data over local area networks. A study [138] focused on decentralised data for the accessing of EHRs and PHRs with high performance; these data were saved in distributed data centres. Another study enhanced a healthcare information system to provide high-quality information at any time and any place [139]. The second area, that is, tele-expertise between professionals, focused on exchanging experiences between physicians or professionals to share knowledge and formulate proper decisions for diagnosis and treatment [15, 140-143].

Finally, the sixth domain is the remote monitoring (telemonitoring). Further investigations regarding this domain are presented in the following subsection.

Remote monitoring (telemonitoring) In remote monitoring, the medical professional monitors the emergency cases of patients remotely, as well as provides real-time data analysis and instant feedback to patients $[15,23]$. The domain of remote monitoring includes studies that are categorised in three areas: patient triage, provision of services to the patient and patient prioritisation.

The first area is patient triage, that is, categorising patients on the basis of their emergency cases. Some studies focused on inside ED triage, which is discussed by [144-146], whereas other studies focused on outside ED triage, which is discussed in [147-150].

The second area contributes to remote healthcare service provision. Several studies have focused on an alert emergency service that alarms a caregiver to provide fast response in case when vital signs become irregular [151-156]. Many studies provided services, such as suggestions, tips, drug prescriptions, recommendations and telehealth consultation [157-161]. Some studies recommended on selecting nearby and suitable hospitals or physicians according to patients' conditions $[162,163]$. A study focused on providing healthcare services and treatment while travelling vehicles on the fly [164]. Another study presented the existence of first-aid services in an ambulance [165]. Moreover, another study introduced a new eldercare service that connects the clinic of a remote physical therapist and the home of an elderly
[166]. Finally, [167] presented an approach for managing the prescribed medication for patients by notifying them about drugs and doses.

Patient prioritisation The third area is patient prioritisation. This section addresses patient prioritisation by serverbased (Tier 3) telemedicine application, indicating that the prioritisation of patients should be appropriately arranged to determine the necessary treatment and to transport them to hospitals according to the patients' condition. In the literature, telemedicine application has been studying on patient prioritisation. This study mainly focused on the remote monitoring of patients, as well as on the proposed application based on android and using IoT. This application includes two stages: Firstly, vital signs are gathered from various sensors, and then it categorises patient status. Secondly, the application sends the gathered vital signs through LTEFemto Cell networks to healthcare centres for patient prioritisation [168]. Another research attempted to develop a system that is based on optimisation using multiswarm PSO technique and can handle some healthcare issues, such as the delivery of services to numerous patients, difficulty in storing large data because of unavailability of servers and data requests that are uncategorised and not enhanced by patient prioritisation [169]. Another research utilised 'MSHA' to present a system of telemonitoring based on integration sensory sources (BP, ECG and $\mathrm{SpO} 2$ ) and non-sensory source (text inputs). The research tried to enhance the efficiency of healthcare scalability by assisting the remote prioritisation and triaging of the heart chronic diseases of patients [23]. Lastly, another study [26] proposed a methodology to enhance decision-making process for prioritising patients with CHD through telemedicine.

Healthcare service concerns in telemedicine This section introduces an overview of healthcare service concerns in telemedicine. Further healthcare services are apparently required, particularly services supplied from outside of hospitals [170]. Chronic diseases (e.g., cardiovascular diseases, cancer and diabetes) are critical matters for healthcare services, given that these diseases embody one of the first causes of mortality, morbidity and disability [171]. Several challenges occur in healthcare service provision for telemonitoring systems [23, 31]. However, this study focused on the challenges related to scalability. The challenges in healthcare service in relation to scalability problem are presented in taxonomy shown in Fig. 4.

Telemonitoring systems face many serious issues, one of which is patient prioritisation in healthcare service scalability [23]. The increasing demand for healthcare service has resulted in the essential need to prioritise patients $[172,173]$. 


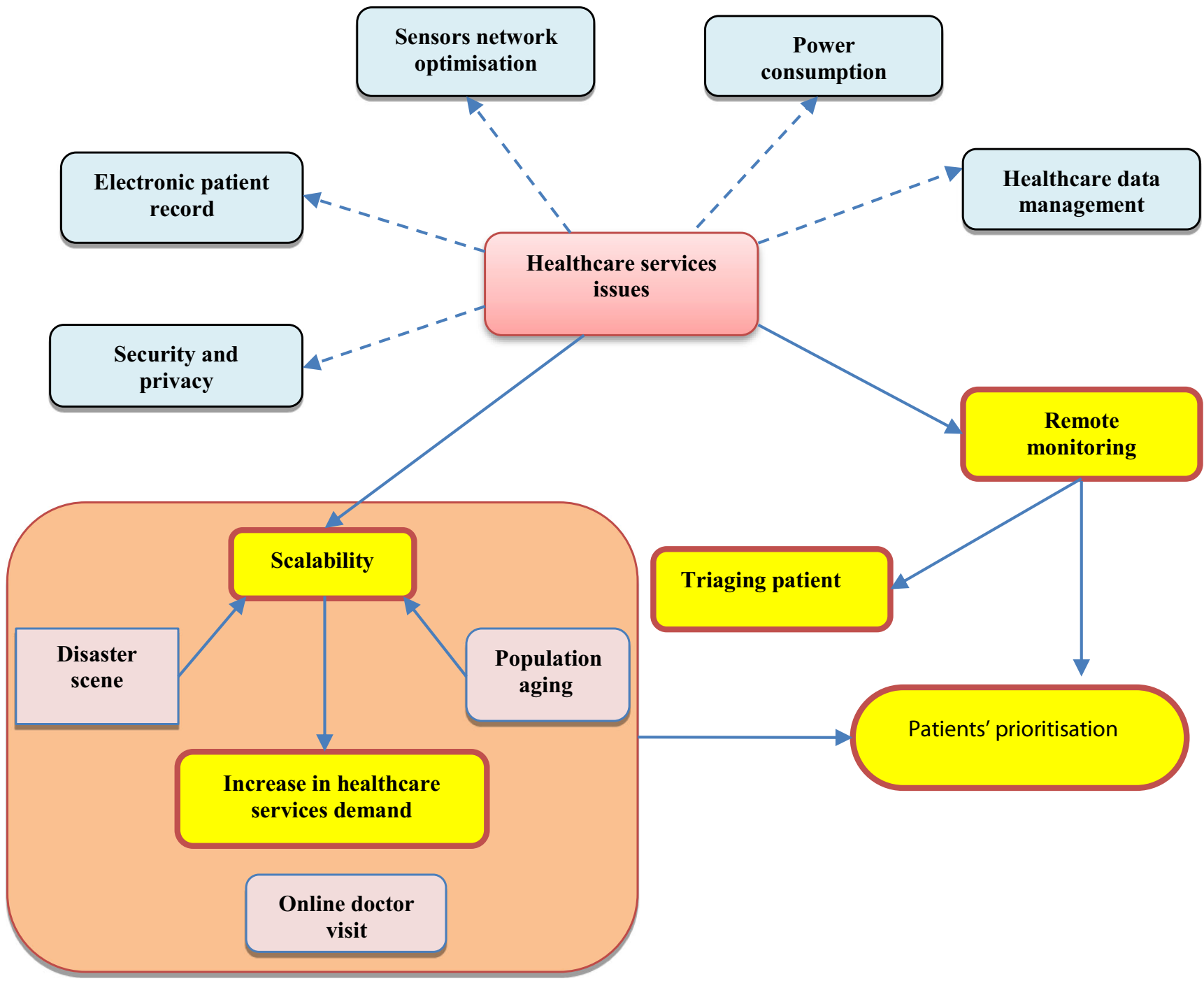

Fig. 4 Taxonomy of healthcare service concerns and scalability problems

Patient prioritisation in healthcare services scalability concerns One of the main concerns in providing healthcare service is scalability, particularly in the processes of patient prioritisation [23]. With the growing demand for healthcare service, medical services that are highly effective and scalable are seriously needed $[172,173]$. Many research have been conducted to enhance the prioritisation of patients in telemedicine context, as well as resolve the scalability issues [23]. The dilemma of the growing number of elderly people who need timely and effective telemedicine services has been discussed in literature. The increase in the number of patients is estimated to happen in various cases, such as disasters and ageing population, as illustrated in Fig. 5 and presented in the subsections below.

Ageing population Healthcare services suffer from massive challenges due to the increase in the number of patients [28, $174,175]$. Healthcare professionals have to use any advanced system effectively to meet the increasing system demand [176]. The key reasons of the continuous growth of older generation sets are current demographic changes [177] that cause persistent and earnest problems, such as incidence of ageing diseases and social and economic burdens [177-179]. A study found an increase in the number of people aged 45 years or more by $11 \%$ in the last contract [180]; the percentage of visits by physicians in this age range for the same duration also increased by $26 \%$.

As the number of patients in healthcare increases, the expenditure of patients for healthcare services also increases [180]. Therefore, the increase in the number of elderly patients is a challenge in telemedicine systems [23]. Globally, the society and the healthcare system are loaded with burdens derived from the ongoing ageing population problems [177-179]. By 2030, 13\% of the world total population is expected to be assigned to around one billion people aged 65 years or older [181]. 
Fig. 5 Problems that cause the increase in users' request in RHMS

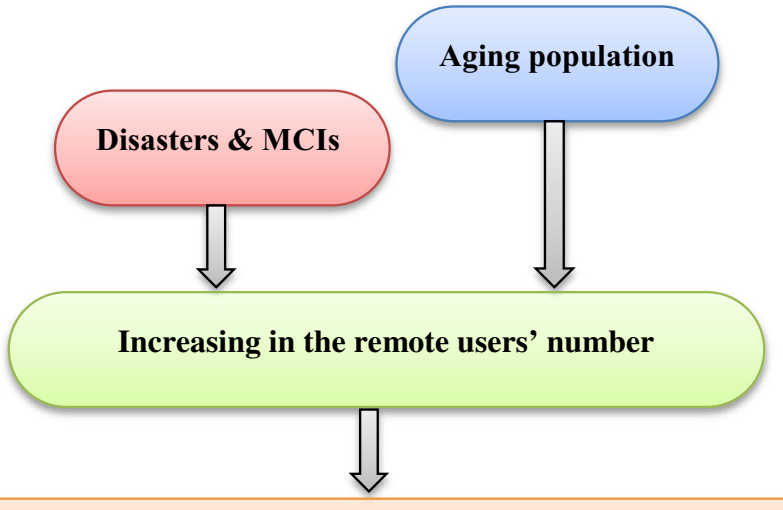

Increasing in the remote users' requests for the healthcare services
Numerous problems in chronic diseases are related to the momentary rise in ageing population and directly influence medical healthcare expenses [182-184]. The increase of chronic disease cases along with the increase of ageing population (e.g., heart failure, hypertension and diabetes) makes the healthcare management a serious issue for health systems worldwide [182]. According to the Medicare and Medicaid Services Centre [180], healthcare expenditure in the USA in 1970 was around $\$ 75$ billion, and it increased more to than $\$ 4.3$ trillion in 2018, as shown in Fig. 6.

Challenge to global healthcare systems in terms of care delivery quality $[180,184]$. Growth in the number of senior citizens leads to the growth in the number of elderly patients [23]. Therefore, researchers have begun to examine how to improve healthcare service provision systems in response to the ageing population problems. They have been investigating and discussing how to enhance healthcare service provision systems responding to the ageing-population issue. A system that could precisely identify heartbeat for elderly patient monitoring was proposed in [185].

Disasters and MCls Disasters cause injuries to a considerable number of people yearly. However, the provision of medical services for injured persons remains a concern [41]. In the study of [186] a system that could be applied to monitor the pulse rate and $\mathrm{SpO} 2$ of casualties was proposed. Furthermore, in [187], researchers proposed the use of a monitoring system for the pulse rate and ECG of casualties. These systems can be

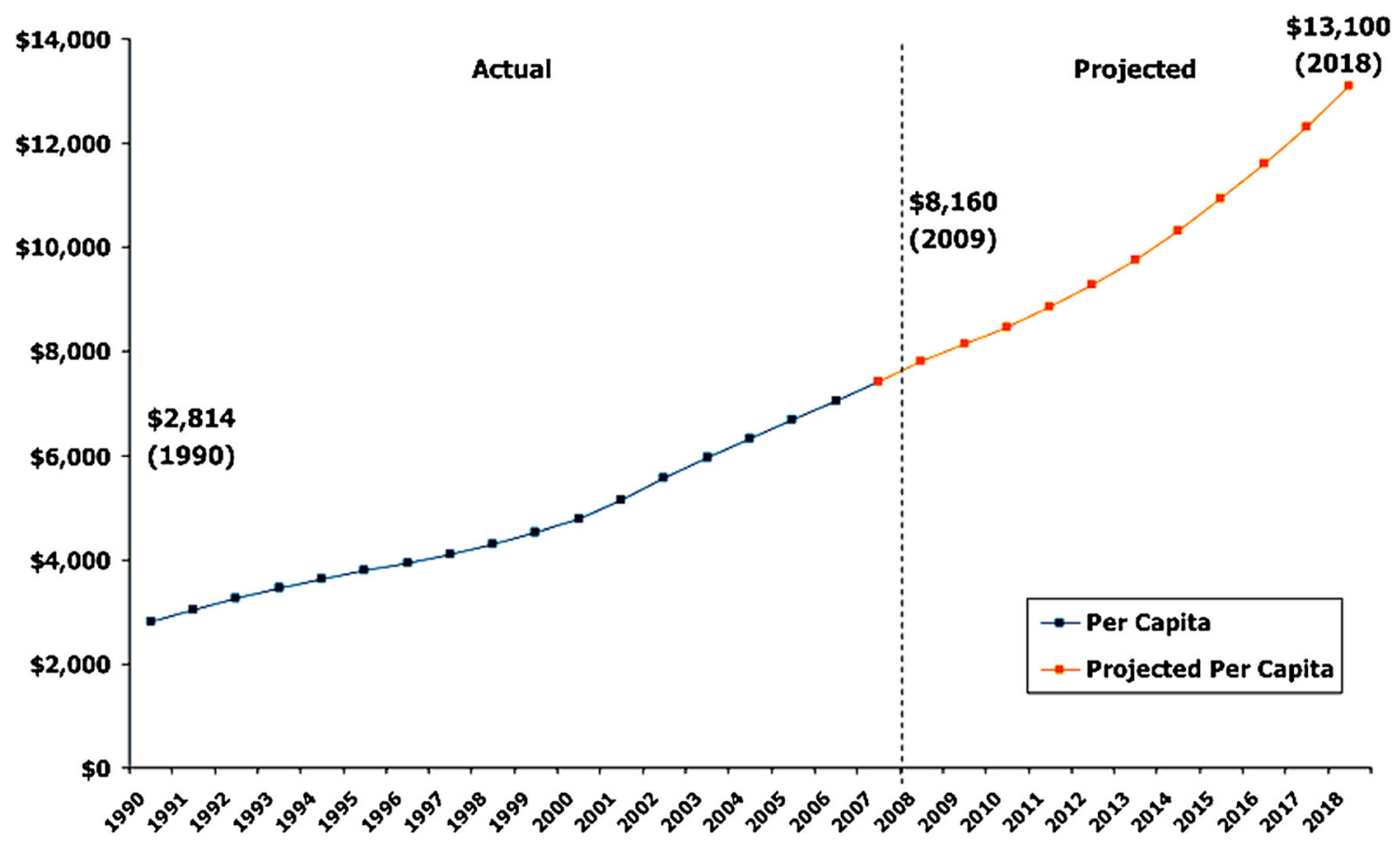

Fig. 6 National healthcare expenditure per capita in the USA [180] 
used to transfer data to the server side. In such systems, rescue commanders can monitor casualties in real time. However, the casualties are not prioritised accordingly. In [188], the researchers demonstrated how various characteristics for an emergency scenario influence protocol behaviour; they compared the efficiency of common opportunistic routing protocols by simulating disaster scenarios. The study of [189] proposed the network for queuing for the health condition of victims in the disaster aftermath. An electronic triage system was also proposed by [186]. This system is composed of two types, namely, the electronic triage (E-Triage) tags and ETriage server. The attached E-Triage tags, which support the monitoring of vital signs for casualties, enables officers to identify a casualty whose physiological condition is drastically changed and requires a rapid response. However, a detailed triage mode is insufficient in mass casualties or in cases in which a triage officer is late. Therefore, identifying new risk evaluation and classification systems enhances intervention and assists patients, caregivers and their providers in disaster preparation [190]. In MCI, patients under remote monitoring might face disruption with the support services required [191]. The capacities of healthcare systems to immediately provide elective healthcare services to causalities are limited; one way to solve such problem is to prioritise the patients remotely to deal with the crowding in case of scalability concerns [23, 192].

Critical review and analysis This section shows a critical review and deep analysis of the telemedicine application review in terms of patients' prioritisation. Physicians or nurses have no time to search huge records and determine the vital signs of patients, particularly when the number of patients with emergency conditions is considerable; therefore, a support is needed for them to focus their attention and efforts to delivering excellent care and discerning which patients need immediate care and which patients can wait. A prioritisation process is utilised in telemedicine environment to rank patients and arrange them according to severity and facilitate the process of distinguishing patients with the most urgent condition.

Based on the systematic literature review, the studies by $[23,26]$ are considered the most relevant in this area. Such studies addressed patient prioritisation in a remote environment and identified those who had CHD. However, such prioritisation solution can only address prioritisation on patients with single chronic disease. In [23], the patients were prioritised by using a priority code (PC) with a range of 0 100 . However, only patients with this range can be prioritised, whereas those with the same PC value were sorted in a descending order by using the first come first serve (FCFS) approach. Moreover, FCFS cannot be used in reality, given that some patients may have more urgent cases than those who arrived earlier [193, 194].
Meanwhile, the study of [26] presented a new approach for prioritising the large-scale data of patients with CHDs; this approach uses body sensors during disasters and peak seasons. The patients in this study were scored according to the MCDM techniques, namely, integrated analytic hierarchy process (AHP) and TOPSIS. However, one major problem in this study is that the proposed approach can only prioritise patients with a single chronic disease; therefore, this approach cannot determine the emergency degree of each chronic disease individually to accurately prioritise patients with MCDs.

This research has not come across any other research that has exclusively presented a solution for remote prioritisation in a large scale of patients with MCDs, especially in the server side of telemedicine applications (Tier 3). Such issue, if handled and addressed, can save lives, among other advantages.

Furthermore, [26] recommended the adoption of a decision-making platform for different chronic diseases (myocardial infarction, diabetes and high or low BP). This platform can be used for patient prioritisation, given the different diseases and emergency levels of each patient. As mentioned, the present study aims to introduce ways to improve the prioritisation process for patients with MCDs in the server (Tier 3) by designing and refinement to accommodate patient's requests and strictly prioritise and rank patients with emergency conditions. Finally, in line with the relevant study in the remote monitoring environment, the MCDs were considered as proofs of concept. Further investigation on RHM through telemedicine and the case study is demonstrated in the following section.

\section{MCDs in RHMS}

Patients may experience various chronic diseases, including diabetes, chronic BP disease, and CHD. Chronic diseases have become a growing serious concern in E-Systems of healthcare worldwide. For instance, the clinical expenses of chronic diseases in the USA are projected to reach $80 \%$ of the total medical costs, and more than 150 million people may have chronic diseases by 2020 [195]. Moreover, health systems and individuals bear huge burdens by chronic diseases due to the frequent visits of patients, who have chronic diseases, to the ED that are frequently unscheduled, as well as lengthy admissions in hospital $[173,196]$.

Healthcare researchers and practitioners have been working on health monitoring applications out-of-hospital, particularly in the home environment, for a long time. Telemedicine requires continuous monitoring of the data relevant to physiological conditions; these data can be used to manage chronic diseases [197]. Patients whose homes are situated far from hospitals and who utilise telemedicine may have various chronic cases, such as chronic BP, CHD and diabetes [198, 199]. Thus, we present a scope for two popular chronic 
diseases from two perspectives, namely, medical and chronic diseases, as presented in the next subsections.

\section{CHD}

Around 12 million people die around the world annually due to CHDs [195]. CHDs have several kinds, and disease symptoms can appear in patients. For instance, 'cardiac arrhythmia' is an emergency case that can be life threatening and can lead to sudden death and cardiac arrest. According to the American Heart Association (2010), arrhythmia caused the death of around 55\% of patients who had heart disease [198]. Serious cases of arrhythmia, such as fibrillation or ventricular tachycardia are common results of vortex-like re-entrant electric waves in the cardiac tissue. The timely diagnosis of CHDs is crucially needed in the medical field due to the impact of heart disease to the health and working performance of patients, particularly elderly patients [195]. Telemedicine plays a pivotal role in the delivery of efficient healthcare to patients with various cardiologic diseases [200]. Numerous research discuss the involvement of remote monitoring in the management of cardiac diseases and cardiac home treatments, demonstrating that remote monitoring is suitable in terms of cost effectiveness for the same health outcome. Moreover, telemedicine methods contribute to chronic heart failure care [201]. In-home telemonitoring also reduces mortality and hospitalisation rate [201].

\section{Chronic BP diseases}

Around $40 \%$ of people worldwide, aged over 25 years, have hypertension [202]. In general, low-income countries have weaker dominance of hypertension (35\%) than other countries (40\%) [202, 203]. Moreover, the largest percentage of people with hypertension who are uncontrolled, untreated and undiagnosed is higher in low-income countries due to poor health applications and systems than in high-income countries. This rise in the distribution of hypertension is due to population growth, ageing and behavioural risk factors [204]. In summary, deaths caused by hypertension increases when no appropriate action is taken. Rapid detection, suitable treatment and superior hypertension management have important health and economic advantages. In [205], 4494 elderly people who lived in Singapore have hypertension. People who live alone have a higher rate of untreated hypertension (37.3\%) than those who do not. In [206], the researchers found that hypertension risk in elderly people who live alone is greater than that in elderly people who are married or living with others.

One common gradual path in managing hypertension is for patients living at home to measure their own $\mathrm{BP}$ and send the measurement results in real time to their caregivers. Research has clarified that monitoring $\mathrm{BP}$ at home is as dependable and authoritative as ambulatory BP tracking [207]. This research suggests a telehealth system that transmits BP data to a server and provides a messaging function, which sends daily reminders to patients. Moreover, in this research, users had a positive perception of the usefulness and usability of the telehealth system. The use of algorithms for health monitoring through BP sensors has been proposed in many studies [23, 208-210]. Finally, clinical guidelines, as well as programs for disease management, cover only single diseases, and clinical studies often excludes people with MCDs [211]. However, MCDs (e.g., CHD, high BP and low BP) have been set as a case study in this research, despite being the leading causes of death worldwide. The sources used to measure vital signs and provided to patients with MCDs are further described and summarised in the next section.

\section{Sources used to measure patients' medical vital signs}

The continuous monitoring of health in WBAN needs the sensors of the system to work at all times [212]. To measure the vital signs of patients, healthcare professionals use many medical devices. In telemedicine applications, sensors play an important role, and patients may need to use various kinds of sensors [184, 213]; The kind and number of sensors required for patient monitoring vary among diseases. In [23], the researchers stated that four medical sources related to CHD must be identified and validated; three of these sensors are utilised as signal sources, and one, as a text source. A set of heterogeneous sources relevant to CHD can be applied in one healthcare platform, which is significant in healthcare monitoring [23, 214]. Table 1 illustrates the brief description of four related medical sources that can be used to triage patients with various chronic diseases.

As shown in Table 1, several sensors, sources and text (heterogeneous sources) are needed in this study. These sources present the diagnosis and reflect the medical signs and symptoms of patients with MCDs.

In conclusion, ECG, SPO2 and BP sensors are important, and they must be employed for CHD. Furthermore, in remote healthcare monitoring, non-sensory data are necessary [23], so that text data can be used as another medical source for the same disease, that is, CHD. Meanwhile, in chronic BP disease, sensors for systolic and diastolic BP can be used in this research to measure the emergency level of patients to enhance the accuracy of triage and healthcare services for patients who have MCDs. Moreover, connections between complaints and vital signs are operationalised as the changing relative significance of vital signs [218]. Hence, each chronic disease (heterogeneous data) has different sources; sensors for MCDs and text should be engaged in this research. Vital signs, such as $\mathrm{SpO} 2$ and ECG, are extremely important to the triage process because they provide an objective complement to the triage decision and optimise inter-rater consistency [219]. For each source of these diseases, classifying the patients and their 
Table 1 Description of relevant medical sources used in monitoring patients

\begin{tabular}{lll}
\hline Source & Description & References \\
\hline ECG & $\begin{array}{l}\text { Measure the electrical representation of the contractile activity of } \\
\text { the heart over time. An electrical representation of contractile } \\
\text { activity is used for the short-term assessment of cardiovascular } \\
\text { diseases, especially for people with chronic heart problems. } \\
\text { The pulse oximeter is used to measure the blood oxygen saturation } \\
\text { level of the patient. } \\
\text { Measure the physiological data of the systolic and diastolic blood } \\
\text { pressure of the patient. }\end{array}$ & {$[23,201,215,216]$.} \\
BP 2 & $\begin{array}{l}\text { Non-sensory measurements are used by triage nurses in the hospital } \\
\text { (ED) to prioritise the patients according to several categories, }\end{array}$ & {$[23,201,216]$.} \\
Text & such as chest pain. & {$[23,215,217]$.} \\
& & \\
\hline
\end{tabular}

diagnostic information by using the triage process is needed. Triage protocols can be used in determining the treatment priority of patients depending on the severity of their situations [146].

\section{Concern for prioritisation process}

Recently, patient prioritisation in terms of MCDs has some shortcomings, given that it still confronts problems and issues related to making multiple decisions. Patient prioritisation with MCDs cannot be solved in existing methods because of various diseases affecting each other. The prioritisation of MCDs in telemedicine applications is a difficult and challenging task because each patient has a multi-group triage of different diseases for evaluating their emergency status. The multi-group triage levels vary among patients on the basis of emergency status. For instance, data variation in multigroup triage for each patient occurs when patient A has a risk level for heart disease, urgent level for high BP and normal level for low BP, whereas patient B has an urgent level for heart disease, normal level for high BP and risk level for low BP. In this case, patient prioritisation is difficult due to the several levels in each patient. Patient A can be prioritised at the highest level on the basis of heart disease (first disease). By contrast, patient $\mathrm{B}$ can be prioritised at the highest level on the basis of low BP (third disease). In such case, a confusing question arises, that is, which patient will be prioritised first?

Fig. 7 MCDM methods

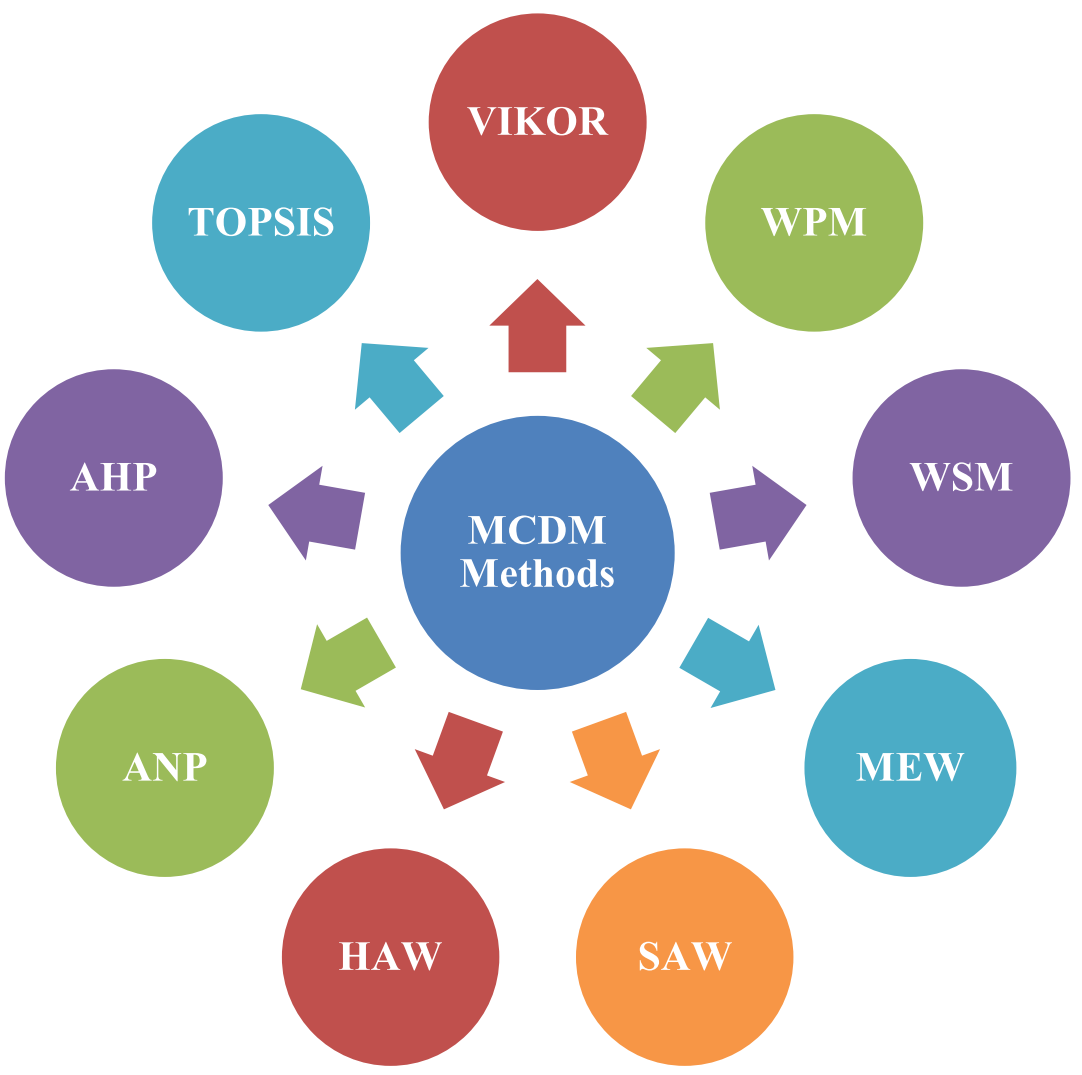


Table 2 Advantages and disadvantage of MCDM techniques

\begin{tabular}{|c|c|c|}
\hline Method & Advantages & Disadvantage \\
\hline HAW \& WSM & - Easily understood and used & $\begin{array}{l}\text { - Arbitrarily assigned attribute weights } \\
\text { - Difficult adoption with numerous criteria } \\
\text { - Use of common numerical scaling in calculating final score }\end{array}$ \\
\hline WPM \& MEW & $\begin{array}{l}\text { - Capability of eliminating any element to be measured } \\
\text { - Use of proportional (rather than real or actual) values }\end{array}$ & - Incapability of providing any solution with equal DM weight \\
\hline SAW & $\begin{array}{l}\text { - Consideration of all criteria/attribute } \\
\text { - Simple calculation } \\
\text { - Intuitive DM }\end{array}$ & $\begin{array}{l}\text { - Need for positive and maximum values for all criteria } \\
\text { - Common incapability of discovering real situation }\end{array}$ \\
\hline AHP & $\begin{array}{l}\text { - Enablement of DMs to structure decision-making problems into } \\
\text { hierarchy trees } \\
\text { - Facilitation of understanding of problems } \\
\text { - Time-consuming support caused by large number of pairwise } \\
\text { comparisons and need for mathematical calculations, which } \\
\text { increase with number of attributes or alternatives } \\
\text { - Substantial restriction imposed by human capacity for information } \\
\text { processing ( } 7 \pm 2 \text { is regarded as comparison ceiling) }\end{array}$ & $\begin{array}{l}\text { - Dependency of scoring and ranking on alternatives } \\
\text { considered for evaluation } \\
\text { - Potential change in final ranking caused by removal or } \\
\text { addition of alternatives (rank reversal problem) }\end{array}$ \\
\hline ANP & $\begin{array}{l}\text { - Provision of full understanding of importance level that can be } \\
\text { assumed by an attribute regarding its correlation with other } \\
\text { attributes } \\
\text { - Enablement of measurement of judgments' consistency, which } \\
\text { cannot be evaluated when weights are specified by compromise } \\
\text { - Assistance in specifying weights by separation of problem into } \\
\text { small parts such that experts can have manageable discussion } \\
\text { because only two attributes are compared in specifying } \\
\text { judgments }\end{array}$ & $\begin{array}{l}\text { - Complexity of offering proper network structure among } \\
\text { attributes even for experts (Different structures lead to } \\
\text { varying results.) } \\
\text { - Need for formation of super matrix to be pairwise } \\
\text { comparison of all attributes with all other attributes } \\
\text { (complex and unnatural process) }\end{array}$ \\
\hline $\begin{array}{l}\text { TOPSIS } \\
\quad \& \text { VIKOR }\end{array}$ & $\begin{array}{l}\text { - Significant approaches to solving real-world problems } \\
\text { - Application in discretising alternative challenges } \\
\text { - Capability of immediately recognising proper alternative } \\
\text { - Decrease in number of required pairwise comparisons, with } \\
\text { capacity limitation not necessarily controlling the process } \\
\text { - Useful when alternatives and attributes are numerous and when } \\
\text { quantitative or objective data are available } \\
\text { - Basis in aggregating function representing 'closeness to the ideal', } \\
\text { which originates from compromise programming method }\end{array}$ & $\begin{array}{l}\text { - TOPSIS and VIKOR include the lack of provision to weigh } \\
\text { elicitation and check the consistency of judgments } \\
\text { - TOPSIS does not consider the relative importance of } \\
\text { distances. }\end{array}$ \\
\hline
\end{tabular}

Finally, the proposed work aims to provide prioritisation for patients with MCDs patients, with simultaneous consideration of the triage levels from MCDs, thereby requiring multiple decision-making in identifying the emergency degree of each chronic disease for each patient. In such case, all patients have an available alternative for the decision makers. Thus, the prioritisation of patients with MCDs involves complex multiple decision-making problems. Hence, multiple-criteria decision analysis is essentially needed to address this situation. The next section presents the suitability of the existing MCDM methods to solve the complexity of the prioritisation process for patients with MCDs.

\section{MCDM: an overview}

In this section, the definition, advantages, challenges and techniques of MCDM are reviewed. The condition of the art of MCDM techniques in the healthcare application is also discussed.

\section{MCDM: definition and importance}

The book by Keeney and Raiffa [220] defines MCDM as 'an extension of decision theory that covers any decision with multiple objectives. A methodology for assessing alternatives on individual, often conflicting criteria, and combining them into one overall appraisal...' Another important definition was provided by Stewart and Belton [221], that is, 'an umbrella term to describe a collection of formal approaches that seek to take explicit account of multiple criteria in helping individuals or groups explore decisions that matter'.

MCDM is considered one of the common techniques in decision-making, and it handles the problems depending on available criteria [222-225]. MCDM involves structuring, planning and solving decision problems by utilising numerous criteria [222, 226, 227]. The purpose of MCDM is to support decision-makers to solve problems with numerous criteria and alternatives [228-230]. It uses a group of qualitative and quantitative methods to overcome complex decision cases, considering multiple criteria, which might be conflicting [231, 232]. 
MCDM can enhance the quality of decisions; it provides the decision-makers to choose right decisions more effectively and rationally than the traditional processes [233-235]. MCDM includes (A) ranking available alternatives and (B) determining the best alternative from available set of alternatives [236, 237]. Accordingly, the suitable alternative(s) will be scored [238, 239].

MCDM, which was based on 'Health Science Policy Council' recommendation, was first used in healthcare in May 2014. MCDM involves many methods. Currently, it is increasingly used in the healthcare field [45, 240], [241]. The aim of the task force was to present a foundational report on a topic, that is, an MCDM primer, and then focus on initial recommendations on how to best use the MCDM methods to support decision-making in healthcare [242-244].

Thus, the use of MCDM in healthcare has become familiar $[245,246]$. Owing to the different available methods of MCDM, healthcare decisions become accurate, and the ability of decision makers to obtain the best solutions improves [247-249].

\section{Critical analysis for MCDM techniques}

Several MCDM theories have been explored. Figure 7 shows the most commonly used MCDM techniques that use different notations [26, 250-255].

The advantages and disadvantage of the popular MCDM methods are shown in Table 2 [22, 229, 235, 252, 256-262].

According to Table 2, the benefit of TOPSIS and VIKOR are appropriate for situations with many alternatives and attributes. These methods can find the best alternative rapidly and are the best among others. Both methods are also specifically convenient to use when quantitative or objective data are given. However, TOPSIS determines a solution with the shortest distance to the ideal solution and the greatest distance from the negative-ideal solution, but it does not consider the relative importance of these distances [256]. By contrast, VIKOR is functionally related to discrete alternative problems. This technique is one of the most practical routes for solving real-world problems. However, such techniques cannot determine and break the decision into subdecisions to guarantee the accuracy of the prioritisation process and determine the accurate emergency degree for patients with multiple chronic diseases that represent multiple-decision making problems. Even though VIKOR technique produce balance between the overall utility and minimising the regret level, increasing the priority level of patient $n$ over patient $m$ in case patient $n$ is more at risk than patient $m$. Alternative solutions for prioritising patients with MCDs are necessary.

\section{Conclusion}

Telemedicine applications, especially in term of patients' prioritisation, are becoming increasingly popular. These applications open up immense opportunities for real-time patients offering respected medical resources and services. However, relevant research on patients' prioritisation in applications of telemedicine has unaddressed restrictions. Four stages have been presented in this research, which aims to review and analyse the prioritisation of patients with MCDs through telemedicine. In the first stage, no study presented solutions for the remote prioritisation as the ability to rank a large number of patients with MCDs, especially in Tier 3. In the second stage, MCDs (CHD, high BP and low BP) in RHMSs have been identified and discussed as a case study. This stage also summarises sources, which are used to measure the vital signs of patients with MCDs. In the third stage, this paper highlighted that multiple decision-making problems are issues in patients' prioritisation over a telemedicine environment. As a result from this stage, using an MCDM as a solution for complex situations and this issue is essentially required. The fourth stage discussed MCDM techniques. Here, after reviewing the MCDM techniques, we found that current methods cannot be used in the prioritisation process for patients with MCDs. In conclusion, a new decision theory for prioritising patients with MCDs over a telemedicine environment is needed.

Funding This study was funded by Universiti Pendidikan Sultan Idris, under grant FRGS/2016-0066-109-02.

\section{Compliance with ethical standards}

Conflict of interest The authors declare no conflict of interest.

Ethical approval All procedures performed in studies involving human participants are in accordance with the ethical standards of the institutional and/or national research committee and with the 1964 Helsinki declaration and its later amendments or comparable ethical standards.

Informed consent Informed consent was obtained from all individual participants included in the study.

\section{References}

1. Iqbal, S. et al., Real-time-based E-health systems: Design and implementation of a lightweight key management protocol for securing sensitive information of patients. Health Technol. (Berl). 9(2):93-111, 2019.

2. Alanazi, H. O. et al., Meeting the security requirements of electronic medical records in the ERA of high-speed computing. J. Med. Syst. 39(1):165, 2015.

3. Nabi, M. S. A. et al., Suitability of using SOAP protocol to secure electronic medical record databases transmission. Int. J. Pharmacol 6(6):959-964, 2010. 
4. Kiah, M. L. M. et al., An enhanced security solution for electronic medical records based on AES hybrid technique with SOAP/XML and SHA-1. J. Med. Syst. 37(5):9971, 2013.

5. Nabi, M. S. et al., Suitability of adopting S/MIME and OpenPGP email messages protocol to secure electronic medical records. In: Second international conference on future generation communication technologies (FGCT 2013), 2013, 93-97.

6. Alanazi, H. O. et al., Securing electronic medical records transmissions over unsecured communications: An overview for better medical governance. Journal of Medicinal Plants Research 4(19): 2059-2074, 2010.

7. Nabi, M. S. A., Kiah, M. M., Zaidan, B. B., Zaidan, A. A., and Alam, G. M., Suitability of SOAP protocol in securing transmissions of EMR database. Int. J. Pharmacol 6(6):959-964, 2010.

8. Rogove, H. J., McArthur, D., Demaerschalk, B. M., and Vespa, P. M., Barriers to telemedicine: Survey of current users in acute care units. Telemed. e-Health 18(1):48-53, 2012.

9. Topol, E. J., The patient will see you now: The future of medicine is in your hands. In: Tantor Media, 2015.

10. Mohsin, A. et al., Based medical systems for patient's authentication: Towards a new verification secure framework using CIA standard. J. Med. Syst., 2019.

11. Mohsin, A. H. et al., Real-time medical systems based on human biometric steganography: A systematic review. J. Med. Syst. 42(12):245, 2018.

12. Abdulnabi, M. et al., A distributed framework for health information exchange using smartphone technologies. J. Biomed. Inform. 69:230-250, 2017.

13. Zaidan, A. A. et al., Challenges, alternatives, and paths to sustainability: Better public health promotion using social networking pages as key tools. J. Med. Syst. 39(2):7, Feb. 2015.

14. Zaidan, B. B. et al., A security framework for nationwide health information exchange based on telehealth strategy. J. Med. Syst. 39(5):51, 2015.

15. Doumbouya, M. B., Kamsu-Foguem, B., Kenfack, H., and Foguem, C., A framework for decision making on teleexpertise with traceability of the reasoning. Irbm 36(1):40-51, Feb. 2015.

16. Mohsin, A. et al., Real-time remote health monitoring systems using body sensor information and finger vein biometric verification: A multi-layer systematic review. J. Med. Syst., 2018.

17. Hussain, M. et al., The landscape of research on smartphone medical apps: Coherent taxonomy, motivations, open challenges and recommendations. Comput. methods programs Biomed., 2015.

18. Zaidan, B. B. et al., Impact of data privacy and confidentiality on developing telemedicine applications: A review participates opinion and expert concerns. Int. J. Pharmacol 7(3):382-387, 2011.

19. Gilpin, R., and Gilpin, J. M., The challenge of global capitalism: The world economy in the 21 st century. Vol. 5. Princeton, NJ: Princeton University Press, 2000.

20. Kiah, M. L. M. et al., MIRASS: Medical informatics research activity support system using information mashup network. Journal of medical systems 38(4):37, 2014.

21. Kiah, M. M. et al., Design and develop a video conferencing framework for real-time telemedicine applications using secure group-based communication architecture. J. Med. Syst., 2014.

22. Kiah, M. L. M. et al., Open source EMR software: Profiling, insights and hands-on analysis. Comput. Methods Programs Biomed. 117(2):360-382, 2014.

23. Salman, O. H., Rasid, M. F. A., Saripan, M. I., and Subramaniam, S. K., Multi-sources data fusion framework for remote triage prioritization in telehealth. J. Med. Syst. 38(9):103, Sep. 2014.

24. Albahri, O. S. et al., Systematic review of real-time remote health monitoring system in triage and priority-based sensor technology: Taxonomy, open challenges, motivation and recommendations. J. Med. Syst. 42(5):80, May 2018.
25. Salman, O. H. et al., Novel methodology for triage and prioritizing using 'big data' patients with chronic heart diseases through telemedicine environmental. Int. J. Inf. Technol. Decis. Mak. 16(05): 1211-1245, Sep. 2017.

26. Kalid, N. et al., Based on real time remote health monitoring systems: A new approach for prioritization 'large scales data' patients with chronic heart diseases using body sensors and communication technology. J. Med. Syst. 42(4):69, Apr. 2018.

27. Albahri, O. S. et al., Fault-tolerant mHealth framework in the context of IoT-based real-time wearable health data sensors. IEEE Access 7:50052-50080, 2019.

28. Seising, R., and Tabacchi, M., Fuzziness and Medicine: Philosophical Reflections and Application Systems in Health Care, vol. 302. Berlin, Heidelberg: Springer Berlin Heidelberg, 2013.

29. Albahri, A. S. et al., Based multiple heterogeneous wearable sensors: A smart real-time health monitoring structured for hospitals distributor. IEEE Access 7:37269-37323, 2019.

30. Acampora, G., Cook, D. J., Rashidi, P., and Vasilakos, A. V., A survey on ambient intelligence in health care. Proc. IEEE. Inst. Electr. Electron. Eng. 101(12):2470-2494, 2013.

31. Rocha, A. et al., Innovations in health care services: The CAALYX system. Int. J. Med. Inform. 82(11):e307-e320, Nov. 2013.

32. Shuwandy, M. L. et al., Sensor-based mHealth authentication for real-time remote healthcare monitoring system: A multilayer systematic review. J. Med. Syst., 2019.

33. Mohsin, A. et al., Based Blockchain-PSO-AES techniques in finger vein biometrics: A novel verification secure framework for patient authentication. Comput. Stand. Interfaces., 2019.

34. Talal, M. et al., Smart home-based IoT for real-time and secure remote health monitoring of triage and priority system using body sensors: Multi-driven systematic review. J. Med. Syst., 2019.

35. Brown, A. M., and Clarke, D. E., Reducing uncertainty in triaging mental health presentations: Examining triage decision-making. Int. Emerg. Nurs. 22(1):47-51, 2014.

36. Román, R. et al., Geographical variations in the benefit of applying a prioritization system for cataract surgery in different regions of Spain. BMC Health Serv. Res. 8:1-10, 2008.

37. Tebé, C., Comas, M., Adam, P., Solans-Domènech, M., Allepuz, A., and Espallargues, M., Impact of a priority system on patients in waiting lists for knee arthroplasty. J. Eval. Clin. Pract. 21(1):9196, 2015.

38. Polk, T. W., Walker, W. P., and Bhatia, D. K., A wireless telemedicine system with extended reporting range and priority messaging. IEEE Dallas Engineering in Medicine and Biology Workshop, DEMBS.:138-141, 2007, 2007.

39. Salman, O. H., Rasid, M. F. A., Saripan, M. I., and Subramaniam, S. K., Multi-sources data fusion framework for remote triage prioritization in telehealth. J. Med. Syst. 38(9):103, Sep. 2014.

40. T. H. Sanders, A. Devergnas, T. Wichmann, and M. A. Clements, "Remote smartphone monitoring for management of Parkinson's Disease," in Proceedings of the 6th International Conference on PErvasive Technologies Related to Assistive Environments, 2013, p. 42.

41. Sakanushi, K. et al., Electronic triage system for continuously monitoring casualties at disaster scenes. J. Ambient Intell. Humaniz. Comput. 4(5):547-558, 2013.

42. Mirkovic, J., Bryhni, H., and Ruland, C., A framework for the development of ubiquitous patient support systems. In: Proceedings of the 6 th international conference on pervasive computing Technologies for Healthcare, 2012, 81-88.

43. Sarkar, P., and Sinha, D., An approach to continuous pervasive Care of Remote Patients Based on priority based assignment of nurse. Lncs 8838:327-338, 2014. 
44. U. S. D. of H. and H. Services, "Multiple chronic conditions-A strategic framework: Optimum health and quality of life for individuals with multiple chronic conditions," Washington, DC US Dep. Heal. Hum. Serv., vol.:2, 2010.

45. Kalid, N. et al., Based real time remote health monitoring systems: A review on patients prioritization and related 'big data' using body sensors information and communication technology. J. Med. Syst. 42(2):30, Feb. 2018.

46. Alsalem, M. A. et al., A review of the automated detection and classification of acute leukaemia: Coherent taxonomy, datasets, validation and performance measurements, motivation, open challenges and recommendations. Comput. Methods Programs Biomed. 158:93-112, May 2018.

47. Zughoul, O. et al., Comprehensive insights into the criteria of student performance in various educational domains. IEEE Access., 2018.

48. Mohsin, A. et al., Blockchain authentication of network applications: Taxonomy, classification, capabilities, open challenges, motivations, recommendations and future directions. Comput. Stand. Interfaces., 2019.

49. Talal, M. et al., Comprehensive review and analysis of antimalware apps for smartphones. Telecommun. Syst., 2019.

50. Alaa, M. et al., A review of smart home applications based on internet of things. J. Netw. Comput. Appl., 2017.

51. Zaidan, A. A. et al., A survey on communication components for IoT-based technologies in smart homes. Telecommunication Systems:1-25, Mar-2018.

52. Zaidan, A. et al., A review on intelligent process for smart home applications based on IoT: Coherent taxonomy, motivation, open challenges, and recommendations. Artif. Intell. Rev., 2018.

53. Yas, Q. M., Zaidan, A. A., Zaidan, B. B., Hashim, M., and Lim, C. K., A systematic review on smartphone skin cancer apps: Coherent taxonomy, motivations, open challenges and recommendations, and new research direction. Journal of Circuits, Systems and Computers 27(05):1830003, 2018.

54. Bradai, N., and Charfi, E., L. C. Fourati, and L. Kamoun, "Priority consideration in inter-WBAN data scheduling," no. 2016:589600, November 2015.

55. Villalonga, C., Pomares, H., Rojas, I., and Banos, O., MIMUWear: Ontology-based sensor selection for real-world wearable activity recognition. Neurocomputing 250 no. 2017, pp. 76-100, Aug. 2017.

56. Ghanavati, S., Abawajy, J., and Izadi, D., A congestion control scheme based on fuzzy logic in wireless body area networks. pp.: 235-242, 2015.

57. Ghanavati, S., Abawajy, J., and Izadi, D., ECG rate control scheme in pervasive health care monitoring system. pp.:22652270, 2016.

58. Rezaee, A. A., Yaghmaee, M. H., and Rahmani, A. M., Optimized congestion management protocol for healthcare wireless sensor networks. Wirel. Pers. Commun. 75(1):11-34, 2014.

59. Rezaee, A. A., Yaghmaee, M. H., Rahmani, A. M., and Mohajerzadeh, A. H., HOCA: Healthcare aware optimized congestion avoidance and control protocol for wireless sensor networks. J. Netw. Comput. Appl. 37:216-228, 2014.

60. Almadani, B., Saeed, B., and Alroubaiy, A., Healthcare systems integration using real time publish. Comput. Electr. Eng. 50:6778, 2016.

61. Claudio, D., Kremer, G. E. O., Bravo-Llerena, W., and Freivalds, A., A dynamic multi-attribute utility theory-based decision support system for patient prioritization in the emergency department. IIE Trans. Healthc. Syst. Eng. 4(1):1-15, 2014.

62. S. Gambhir, DWBAN : Dynamic priority based WBAN architecture for healthcare system, pp. 0-6, 2016.
63. Zhao, Y., and Kerkhoff, H. G., Design of an Embedded Health Monitoring Infrastructure for accessing multi-processor SoC degradation. pp.:154-160, 2014.

64. Shah, M. A., Kim, J., Khadra, M. H., Feng, D., and Fellow, I., Home area network for optimizing telehealth services- empirical simulation analysis. pp.:1370-1373, 2014.

65. Lin, D. et al., Admission control over internet of vehicles attached with medical sensors for ubiquitous healthcare applications. IEEE J. Biomed. Heal. INFORMATICS 20(4):1195-1204, 2016.

66. Hu, L., Zhang, Y., Feng, D., Mehedi, M., Alelaiwi, A., and Alamri, A., Design of QoS-aware multi-level MAC-layer for wireless body area network. J. Med. Syst., 2015.

67. Iftikhar, M., and Ahmad, I., A novel analytical model for provisioning QoS in body area sensor networks. Procedia - Procedia Comput. Sci. 32:900-907, 2014.

68. Iftikhar, M., Al Elaiwi, N., and Aksoy, M. S., The 2 nd international workshop on communications and sensor networks performance analysis of priority queuing model for low power wireless body area networks ( WBANs ). Procedia - Procedia Comput. Sci. 34:518-525, 2014.

69. Sevin, A., C. Bayilmis, and I. Kirbas, "cross-layer medium access protocol for wireless body area," 000:1-12, 2016.

70. Chiang, H.-P., Lai, C.-F., and Huang, Y.-M., A green cloudassisted health monitoring service on wireless body area networks. Inf. Sci. (Ny). 284:118-129, Nov. 2014.

71. Baehr, D., Mckinney, S., Quirk, A., and Harfoush, K., On the practicality of elliptic curve cryptography for medical sensor networks. pp.:41-45, 2014.

72. De, A., Braeken, A., Touhafi, A., and Wouters, K., Secure event logging in sensor networks. Comput. Math. with Appl. 65(5):762$773,2013$.

73. Hedin, D. S., Member, S., Kollmann, D. T., Gibson, P. L., Riehle, T. H., and Seifert, G. J., Distance bounded energy detecting ultrawideband impulse radio secure protocol. pp.:6619-6622, 2014.

74. Soufiene, B. O., Bahattab, A. A., Trad, A., and Youssef, H., Lightweight and confidential data aggregation in healthcare wireless sensor networks. no. November:576-588, 2016, 2015.

75. Rubio, Ó. J., Trigo, J. D., and Alesanco, Á., L. Serrano, and. J. García, "Analysis of ISO / IEEE 11073 built-in security and its potential IHE-based extensibility," 60:270-285, 2016.

76. T. Benmansour, T. Ahmed, and S. Moussaoui, "Performance Evaluation of IEEE 802 . 15 . 6 MAC in monitoring of a cardiac patient," 2016.

77. Fourati, H., Idoudi, H., Val, T., Van Den Bossche, A., and Saidane, L. A., Performance evaluation of IEEE 802.15.6 CSMA/CAbased CANet WBAN. In: 2015 IEEE/ACS 12th international conference of computer systems and applications (AICCSA), 2015, $1-7$.

78. Radhakrishnan, S., Duvvuru, A., and Kamarthi, S. V., Investigating discrete event simulation method to assess the effectiveness of wearable health monitoring devices. Procedia Econ. Financ. 11:838-856, 2014.

79. Meizoso, J. P. et al., Evaluation of miniature wireless vital signs monitor in a trauma intensive care unit. Mil. Med. 181(c):199 204, 2017.

80. Ru Kong, C., and Chen, W. Y., B. Yang, and X. Guan, "Data priority based slot allocation for Wireless Body Area Networks," in 2013 International Conference on Wireless Communications and Signal Processing:1-6, 2013

81. Misra, S., and Sarkar, S., Priority-based time-slot allocation in wireless body area networks during medical emergency situations: An evolutionary game-theoretic perspective. IEEE J. Biomed. Heal. Informatics 19(2):541-548, Mar. 2015.

82. Puri, T., Energy efficient QoS aware MAC layer time slot allocation scheme for WBASN. pp.:966-972, 2015. 
83. Ben, H. et al., A priority based cross layer routing protocol for healthcare applications. Ad Hoc Networks 42:1-18, 2015.

84. Bradai, N., L. C. Fourati, and L. Kamoun, "Ad Hoc Networks WBAN data scheduling and aggregation under WBAN / WLAN healthcare network," 25:251-262, 2015.

85. Gündoğdu, K., and Çalhan, A., An implementation of wireless body area networks for improving priority data transmission delay. J. Med. Syst. 40(3):75, Mar. 2016

86. Kaur, J., and Scholar, P. G., Priority based congestion avoidance hybrid scheme for wireless sensor network, no. September, pp., 2015, 4-5.

87. Kim, R. H., and Kim, P. S., An effect of delay reduced MAC protocol for WBAN based medical signal monitoring. pp.:434 437, 2015.

88. Ahmed, A., and Al Mamoon, I., Architecture and communication protocols for cognitive radio network enabled hospital. pp.:170 174, 2015.

89. Rezvani, S., A. Ghorashi, "context aware and channel-based resource allocation for wireless body area networks," vol. 3, no. 2013:16-25, November 2012.

90. Ren, J., Wu, G., Li, X., Pirozmand, P., and Obaidat, M. S., Probabilistic response-time analysis for real-time systems in body area sensor networks, no. may, pp., 2015, 2145-2166.

91. Hussain, M. et al., Conceptual framework for the security of mobile health applications on android platform. Telematics and Informatics, Mar-2018.

92. Hussain, M., A security framework for mHealth apps on android platform. Comput. Secur., 2018.

93. Alnanih, R., Ormandjieva, O., and Radhakrishnan, T., Contextbased and rule-based adaptation of Mobile user interfaces in mHealth. Procedia Comput. Sci. 21:390-397, 2013.

94. Fratini, A., and Caleffi, M., Telematics and informatics medical emergency alarm dissemination in urban environments. Telemat. Informatics 31(3):511-517, 2014.

95. O. Boursalie, R. Samavi, and T. E. Doyle, M4CVD: Mobile machine learning model for monitoring cardiovascular disease, Procedia Comput. Sci., vol. 63, no. Icth, pp. 384-391, 2015.

96. M. Fezari, R. Rasras, and I. M. M. El Emary, Ambulatory health monitoring system using wireless sensors node, Procedia Comput. Sci., vol. 65, no. Iccmit, pp. 86-94, 2015.

97. Villarreal, V., Fontecha, J., Hervas, R., and Bravo, J., Mobile and ubiquitous architecture for the medical control of chronic diseases through the use of intelligent devices: Using the architecture for patients with diabetes. Futur. Gener. Comput. Syst. 34:161-175, May 2014.

98. Sebillo, M., Tortora, G., Tucci, M., Vitiello, G., Ginige, A., and Di Giovanni, P., Combining personal diaries with territorial intelligence to empower diabetic patients. J. Vis. Lang. Comput. 29:114, Aug. 2015.

99. Katib, A., Rao, D., Rao, P., Williams, K., and Grant, J., A prototype of a novel cell phone application for tracking the vaccination coverage of children in rural communities. Comput. Methods Programs Biomed. 122(2):215-228, Nov. 2015.

100. Lwin, M. O. et al., A 21st century approach to tackling dengue: Crowdsourced surveillance, predictive mapping and tailored communication. Acta Trop. 130(1):100-107, Feb. 2014.

101. Bresó, A., Martínez-Miranda, J., Fuster-García, E., and GarcíaGómez, J. M., A novel approach to improve the planning of adaptive and interactive sessions for the treatment of major depression. Int. J. Hum. Comput. Stud. 87:80-91, Mar. 2016.

102. Chakraborty, S., Ghosh, S. K., Jamthe, A., and Agrawala, D. P., Detecting mobility for monitoring patients with Parkinson's disease at home using RSSI in a wireless sensor network. Procedia Comput. Sci. 19:956-961, 2013.

103. Hermens, H., op den Akker, H., Tabak, M., Wijsman, J., and Vollenbroek, M., Personalized coaching systems to support healthy behavior in people with chronic conditions. J. Electromyogr. Kinesiol. 24(6):815-826, Dec. 2014.

104. S. Piotin, A. Benassarou, F. Blanchard, O. Nocent, and E. Bertin, "Abdominal morphometric data acquisition using depth sensors," in 2013 IEEE 15th International Conference on e-Health Networking, Applications and Services (Healthcom 2013), 2013, no. Healthcom, pp. 653-657.

105. Adibi, S., A mobile health network disaster management system. In: 2015 seventh international conference on ubiquitous and future networks, 2015, 424-428.

106. Beck, C., and Georgiou, J., Wearable, multimodal, vitals acquisition unit for intelligent field triage. Healthc. Technol. Lett. 3(3): 189-196, Sep. 2016.

107. Besaleva, L. I., and Weaver, A. C., Mobile electronic triaging for emergency response improvement through crowdsourced and sensor-detected information. In: Proceedings of the 2013 IEEE/ ACM international conference on advances in social networks analysis and mining - ASONAM '13, 2013, 1092-1093.

108. Ganz, A., Schafer, J. M., Tang, J., Yang, Z., Yi, J., and Ciottone, G., Urban search and rescue situational awareness using DIORAMA disaster management system. Procedia Eng. 107: 349-356, 2015.

109. Gunasekaran, S., and Suresh, M., A novel control of disaster protection (NCDP) for pilgrims by pan technology. In: 2014 IEEE 8th international conference on intelligent systems and control (ISCO), 2014, 103-107.

110. A. Renner et al., "RIPPLE: Scalable medical telemetry system for supporting combat rescue," in NAECON 2014 - IEEE National Aerospace and Electronics Conference, 2014, vol. 2015-Febru, pp. 228-232.

111. Ali, R. et al., GUDM: Automatic generation of unified datasets for learning and reasoning in healthcare. Sensors 15(12):1577215798, Jul. 2015.

112. Gaynor, M., and Waterman, J., Design framework for sensors and RFID tags with healthcare applications. Heal. Policy Technol. 5(4):357-369, Dec. 2016.

113. Kim, H.-K., Convergence agent model for developing uhealthcare systems. Futur. Gener. Comput. Syst. 35:39-48, Jun. 2014.

114. Misra, S., and Chatterjee, S., Social choice considerations in cloud-assisted WBAN architecture for post-disaster healthcare: Data aggregation and channelization. Inf. Sci. (Ny). 284:95-117, Nov. 2014.

115. Zhang, K., Liang, X., Baura, M., Lu, R., and Shen, X. S., PHDA: A priority based health data aggregation with privacy preservation for cloud assisted WBANs. Inf. Sci. (Ny) 284:130-141, Nov. 2014

116. Yi, C., Zhao, Z., Cai, J., Lobato de Faria, R., and Zhang, G. M., Priority-aware pricing-based capacity sharing scheme for beyondwireless body area networks. Comput. Networks 98:29-43, Apr. 2016.

117. Yi, C., Alfa, A. S., and Cai, J., An incentive-compatible mechanism for transmission scheduling of delay-sensitive medical packets in E-health networks. IEEE Trans. Mob. Comput. 15(10):2424-2436, 2016.

118. Zhang Yi et al., "Emergency treatment in smart terminal-based ehealthcare networks," in 2015 4th International Conference on Computer Science and Network Technology (ICCSNT), 2015, no. Iccsnt, pp. 1178-1181.

119. Sneha, S., and Varshney, U., A framework for enabling patient monitoring via mobile ad hoc network. Decis. Support Syst. 55(1):218-234, Apr. 2013.

120. Bouakaz, S. et al., CIRDO: Smart companion for helping elderly to live at home for longer. IRBM 35(2):100-108, Apr. 2014.

121. De Backere, F., Bonte, P., Verstichel, S., Ongenae, F., and De Turck, F., The OCarePlatform: A context-aware system to support 
independent living. Comput. Methods Programs Biomed. 140: 111-120, Mar. 2017.

122. Kormanyos, B., and Pataki, B., Multilevel simulation of daily activities: Why and how? In: 2013 IEEE international conference on computational intelligence and virtual environments for measurement systems and applications (CIVEMSA), 2013, 1-6.

123. Rahmani, A. M. et al., Exploiting smart e-health gateways at the edge of healthcare internet-of-things: A fog computing approach. Futur. Gener. Comput. Syst. 78:641-658, Jan. 2018.

124. Varshney, U., A model for improving quality of decisions in mobile health. Decis. Support Syst. 62:66-77, Jun. 2014.

125. Minutolo, A., Esposito, M., and De Pietro, G., Design and validation of a light-weight reasoning system to support remote health monitoring applications. Eng. Appl. Artif. Intell. 41:232-248, May 2015.

126. Ahsanul, S., and Mahfuzul, S., False alarm detection in cyberphysical Systems for Healthcare Applications. AASRI Procedia 5:54-61, 2013

127. N. Pombo, N. Garcia, V. Felizardo, and K. Bousson, "Big data reduction using RBFNN: A predictive model for ECG waveform for eHealth platform integration," in 2014 IEEE 16th International Conference on e-Health Networking, Applications and Services (Healthcom), 2014, no. Ssh, pp. 66-70.

128. Sakr, S., and Elgammal, A., Towards a comprehensive data analytics framework for smart healthcare services. Big Data Res. 4(May):44-58, Jun. 2016

129. Moutacalli, M. T., Marmen, V., Bouzouane, A., and Bouchard, B., Activity pattern mining using temporal relationships in a smart home. In: 2013 IEEE symposium on computational intelligence in healthcare and e-health (CICARE), 2013, 83-87.

130. Bharatula, S., and Meenakshi, M., Design of Cognitive Radio Network for hospital management system. Wirel. Pers. Commun. 90(2):1021-1038, Sep. 2016.

131. V. Vaidehi, M. Vardhini, H. Yogeshwaran, G. Inbasagar, R. Bhargavi, and C. Sweetlin Hemalatha, "Agent based health monitoring of elderly people in indoor environments using wireless sensor networks," Procedia Comput. Sci., vol. 19, no. Ant, pp. 64-71, 2013.

132. Ben Othman, S., Zgaya, H., Hammadi, S., Quilliot, A., Martinot, A., and Renard, J.-M., Agents endowed with uncertainty management behaviors to solve a multiskill healthcare task scheduling. J. Biomed. Inform. 64:25-43, Dec. 2016.

133. Peleg, M. et al., Assessment of a personalized and distributed patient guidance system. Int. J. Med. Inform. 101:108-130, May 2017.

134. Tawfik, H., and Anya, O., Evaluating practice-centered awareness in cross-boundary telehealth decision support systems. Telemat. Informatics 32(3):486-503, Aug. 2015.

135. Tamura, T. et al., Assessment of participant compliance with a web-based home healthcare system for promoting specific health checkups. Biocybern. Biomed. Eng. 34(1):63-69, 2014.

136. Lounis, A., Hadjidj, A., Bouabdallah, A., and Challal, Y., Healing on the cloud: Secure cloud architecture for medical wireless sensor networks. Futur. Gener. Comput. Syst. 55:266-277, Feb. 2016.

137. Saleem, K., Derhab, A., Al-Muhtadi, J., and Shahzad, B., Humanoriented design of secure machine-to-machine communication system for e-healthcare society. Comput. Human Behav. 51:977985, Oct. 2015.

138. Wang, J., Qiu, M., and Guo, B., Enabling real-time information service on telehealth system over cloud-based big data platform. J. Syst. Archit. 72:69-79, Jan. 2017.

139. Nageba, E., Rubel, P., and Fayn, J., Towards an intelligent exploitation of heterogeneous and distributed resources in cooperative environments of eHealth. IRBM 34(1):79-85, Feb. 2013.
140. Sene, A., Kamsu-Foguem, B., and Rumeau, P., Telemedicine framework using case-based reasoning with evidences. Comput. Methods Programs Biomed. 121(1):21-35, Aug. 2015.

141. Doumbouya, M. B., Kamsu-Foguem, B., Kenfack, H., and Foguem, C., Telemedicine using mobile telecommunication: Towards syntactic interoperability in teleexpertise. Telemat. Informatics 31(4):648-659, Nov. 2014.

142. Urovi, V., Jimenez-del-Toro, O., Dubosson, F., Ruiz Torres, A., and Schumacher, M. I., COMPOSE: Using temporal patterns for interpreting wearable sensor data with computer interpretable guidelines. Comput. Biol. Med. 81:24-31, Feb. 2017.

143. Tegegne, T., and van der Weide, T. P. T., Enriching queries with user preferences in healthcare. Inf. Process. Manag. 50(4):599 620, Jul. 2014

144. Moreno, S., Quintero, A., Ochoa, C., Bonfante, M., Villareal, R., and Pestana, J., Remote monitoring system of vital signs for triage and detection of anomalous patient states in the emergency room. In: 2016 XXI symposium on signal processing, images and artificial vision (STSIVA), 2016, 1-5.

145. Dos Santos, J. R. B., Blard, G., Oliveira, A. S. R., and De Carvalho, N. B., Wireless sensor tag and network for improved clinical triage. In: 2015 Euromicro conference on digital system design, 2015, 399-406.

146. Niswar, M. et al., Performance evaluation of ZigBee-based wireless sensor network for monitoring patients' pulse status. In: 2013 international conference on information technology and electrical engineering (ICITEE), 2013, 291-294.

147. Niswar, M. et al., The design of wearable medical device for triaging disaster casualties in developing countries. In: 2015 fifth international conference on digital information processing and communications (ICDIPC), 2015, 207-212.

148. Paulus, A., Czaplik, M., Hirsch, F., Meisen, P., Meisen, T., and Jeschke, S., AUDIME: Augmented disaster medicine. In: Automation, communication and cybernetics in science and engineering 2015/2016. Cham: Springer International Publishing, 2016, 637-644.

149. F. Ullah, A. Khelil, A. A. Sheikh, E. Felemban, and H. M. A. Bojan, "Towards automated self-tagging in emergency health cases," in 2013 IEEE 15th International Conference on e-Health Networking, Applications and Services (Healthcom 2013), 2013, no. Healthcom, pp. 658-663.

150. Rodriguez, D., Heuer, S., Guerra, A., Stork, W., Weber, B., and Eichler, M., Towards automatic sensor-based triage for individual remote monitoring during mass casualty incidents. In: 2014 IEEE international conference on bioinformatics and biomedicine (BIBM), 2014, 544-551.

151. J. Gómez, B. Oviedo, and E. Zhuma, "Patient monitoring system based on internet of things," Procedia Comput. Sci., vol. 83, no. Ant, pp. 90-97, 2016.

152. Hussain, A., Wenbi, R., da Silva, A. L., Nadher, M., and Mudhish, M., Health and emergency-care platform for the elderly and disabled people in the Smart City. J. Syst. Softw. 110:253-263, Dec. 2015

153. Lamprinakos, G. C. et al., An integrated remote monitoring platform towards telehealth and telecare services interoperability. Inf. Sci. (Ny). 308(March):23-37, Jul. 2015.

154. Sung, W.-T., and Chang, K.-Y., Health parameter monitoring via a novel wireless system. Appl. Soft Comput. 22:667-680, Sep. 2014.

155. Zanjal, S. V., and Talmale, G. R., Medicine reminder and monitoring system for secure health using IOT. Procedia Comput. Sci. 78(December):471-476, 2016, 2015.

156. J. Mendes, H. Simões, P. Rosa, N. Costa, C. Rabadão, and A. Pereira, "Secure Low-cost Solution for Elder's eCardio Surveillance," Procedia Comput. Sci., vol. 27, no. Dsai 2013, pp. 46-56, 2014. 
157. AHMED, A., REBEIRO-HARGRAVE, A., NOHARA, Y., KAI, E., HOSSEIN RIPON, Z., and NAKASHIMA, N., Targeting morbidity in unreached communities using portable health clinic system. IEICE Trans. Commun. E97.B(3):540-545, 2014.

158. Ganapathy, K., Priya, B., Priya, B., Dhivya, V. P., and Vaidehi, V., SOA framework for geriatric remote health care using wireless sensor network. Procedia Comput. Sci. 19(Fams):1012-1019, 2013.

159. Miah, S. J., Hasan, J., and Gammack, J. G., On-cloud healthcare clinic: An e-health consultancy approach for remote communities in a developing country. Telemat. Informatics 34(1):311-322, Feb. 2017.

160. P. Moore, A. Thomas, T. Qassem, N. Bessis, and B. Hu, "Monitoring Patients with Mental Disorders," in 2015 9th International Conference on Innovative Mobile and Internet Services in Ubiquitous Computing, 2015, pp. 65-70.

161. Traverso, G. et al., Physiologic status monitoring via the gastrointestinal tract. PLoS One 10(11):e0141666, Nov. 2015.

162. Kovalchuk, S. V., Krotov, E., Smirnov, P. A., Nasonov, D. A., and Yakovlev, A. N., Distributed data-driven platform for urgent decision making in cardiological ambulance control. Futur. Gener. Comput. Syst. 79:144-154, Feb. 2018.

163. Rajkumar, R., and Sriman Narayana Iyengar, N. C., Dynamic integration of Mobile JXTA with cloud computing for emergency rural public health care. Osong Public Heal. Res. Perspect. 4(5): 255-264, Oct. 2013.

164. Kumar, N., Kaur, K., Jindal, A., and Rodrigues, J. J. P. C., Providing healthcare services on-the-fly using multi-player cooperation game theory in internet of vehicles (IoV) environment. Digit. Commun. Networks 1(3):191-203, Aug. 2015.

165. Moretti, S., Cicalò, S., Mazzotti, M., Tralli, V., and Chiani, M., Content/context-aware multiple camera selection and video adaptation for the support of m-health services. Procedia Comput. Sci. 40(C):206-213, 2014.

166. Calyam, P. et al., Synchronous big data analytics for personalized and remote physical therapy. Pervasive Mob. Comput. 28:3-20, Jun. 2016.

167. Teijeiro, T., Félix, P., Presedo, J., and Zamarrón, C., An open platform for the protocolization of home medical supervision. Expert Syst. Appl. 40(7):2607-2614, Jun. 2013.

168. Hindia, M. N., Rahman, T. A., Ojukwu, H., Hanafi, E. B., and Fattouh, A., Enabling remote health-caring utilizing IoT concept over LTE-femtocell networks. PLoS One 11(5):e0155077, May 2016.

169. Ganapathy, K., Vaidehi, V., Kannan, B., and Murugan, H., Hierarchical particle swarm optimization with Ortho-cyclic circles. Expert Syst. Appl. 41(7):3460-3476, Jun. 2014.

170. M. Topaz, "Developing a tool to support decisions on patient prioritization at admission to home health care developing a tool to support decisions on patient prioritization at," 2014.

171. World Health Assembly, Global action plan for the prevention and control of noncommunicable diseases, Geneva World Heal. Organ., 2013.

172. Jeong, S., Youn, C.-H., Shim, E. B., Kim, M., Cho, Y. M., and Peng, L., An integrated healthcare system for personalized chronic disease Care in Home-Hospital Environments. IEEE Trans. Inf. Technol. Biomed. 16(4):572-585, Jul. 2012.

173. Van Dyk, L., A review of telehealth service implementation frameworks. Int. J. Environ. Res. Public Health 11(12):1279-1298, Jan. 2014.

174. Mazomenos, E. B. et al., A low-complexity ECG feature extraction algorithm for mobile healthcare applications. IEEE J. Biomed. Heal. informatics 17(2):459-469, 2013.

175. Wang, X., Gui, Q., Liu, B., Jin, Z., and Chen, Y., Enabling smart personalized healthcare: A hybrid mobile-cloud approach for ECG telemonitoring. IEEE J. Biomed. Heal. informatics 18(3):739745, 2014.

176. Barbera, J. A., and Macintyre, A. G., Medical surge capacity and capability : A management system for integrating medical and health resources during large-scale emergencies. Washington, DC: US Department of Health and Human Services, 2004.

177. Klimova, B., Mobile health devices for aging population groups : A review study, pp. 295-301, 2016.

178. Chung, Y., and Liu, C., Design of a Wireless Sensor Network Platform for tele-homecare. pp.:17156-17175, 2013.

179. Sun, J., and Guo, Y., X. Wang, and Q. Zeng, "mHealth For Aging China : Opportunities and Challenges," 7(1):53-67, 2016.

180. O. Spending, "Trends in health care costs and Spending," 2009.

181. Parekh, A. K., Goodman, R. A., Gordon, C., and Koh, H. K., Managing multiple chronic conditions: A strategic framework for improving health outcomes and quality of life. Public Health Rep. 126(4):460-471, 2011.

182. Palozzi, G., Binci, D., and Appolloni, A., E-health and co-production: Critical drivers for chronic diseases management. In: Service Business Model Innovation in Healthcare and Hospital Management, springer, 2017, 269-296.

183. Sparks, R., Celler, B., and Okugami, C., R. Jayasena, and M. Varnfield, "Telehealth Monitoring of Patients in the Community, " 25(1):37-53, 2016.

184. Touati, F., and Tabish, R., U-healthcare system: State-of-the-art review and challenges. J. Med. Syst. 37(3):9949, 2013.

185. Shih, D.-H., Chiang, H.-S., Lin, B., and Lin, S.-B., An embedded mobile ECG reasoning system for elderly patients. IEEE Trans. Inf. Technol. Biomed. 14(3):854-865, 2010.

186. V. Shnayder, B. Chen, K. Lorincz, T. R. F. Fulford-Jones, and M. Welsh, "Sensor networks for medical care," 2005.

187. Ahn, J., Heo, J., Lim, S., Seo, J., and Kim, W., A study of healthcare system for patient location data based on LBS. In: Consumer electronics, 2008. ICCE 2008. Digest of technical papers. International conference on, 2008, 1-2.

188. MartíN-Campillo, A., Crowcroft, J., Yoneki, E., and Martí, R., Evaluating opportunistic networks in disaster scenarios. J. Netw. Comput. Appl. 36(2):870-880, 2013.

189. Xiang, Y., and Zhuang, J., A medical resource allocation model for serving emergency victims with deteriorating health conditions. Ann. Oper. Res. 236(1):1-20, 2014.

190. Wyte-Lake, T., Claver, M., and Dobalian, A., Assessing patients' disaster preparedness in home-based primary care. Gerontology 62(3):263-274, 2016.

191. R. D. Zane and P. Biddinger, Home Health Patient Assessment Tools: Preparing for Emergency Triage. Abt Associates, 2011.

192. Hansen, P., Hendry, A., Naden, R., Ombler, F., and Stewart, R., A new process for creating points systems for prioritising patients for elective health services. Clin. Gov. An Int. J. 17(3):200-209, 2012.

193. Claudio, D., and Okudan, G. E., Utility function-based patient prioritisation in the emergency department. Eur. J. Ind. Eng. 4(1):59-77, 2010.

194. K. W. Tan, K. W. Tan, and R. Kauffman, "Dynamic queue Management for Hospital Emergency Room Services Emergency Room Services,” 2013.

195. Nguyen, T., Khosravi, A., Creighton, D., and Nahavandi, S., Classification of healthcare data using genetic fuzzy logic system and wavelets. Expert Syst. Appl. 42(4):2184-2197, 2015.

196. W. Mciver, J. Light, M. A. Chowdhury, W. M. Jr, and J. Light, Data association in remote health monitoring systems Data Association in Remote Health Monitoring Systems, no. June, 2012.

197. Patel, S., Park, H., Bonato, P., Chan, L., and Rodgers, M., A review of wearable sensors and systems with application in rehabilitation. J. Neuroeng. Rehabil. 9(1):21, 2012. 
198. M. M. Baig and H. Gholamhosseini, "Smart health monitoring systems : An overview of design and modeling," 2013.

199. Okura, T. et al., The importance of walking for control of blood pressure: Proof using a telemedicine system. Telemed. e-Health 22(12):1019-1023, 2016.

200. Brunetti, N. D. et al., Telemedicine for cardiovascular disease continuum: A position paper from the Italian Society of Cardiology Working Group on Telecardiology and informatics. Int. J. Cardiol. 184:452-458, 2015.

201. Winkler, S. et al., A new telemonitoring system intended for chronic heart failure patients using mobile telephone technology-Feasibility study. Int. J. Cardiol. 153(1):55-58, 2011.

202. Alwan, A., Others, Global status report on noncommunicable diseases 2010. World Health Organization, 2011.

203. W. H. Organization and Others, Global Health Observatory data repository. Causes of death 2008, summary tables. In: Geneva: World Health Organization department of health statistics and informatics, 2008.

204. World Health Organization, World health day. BMJ 1(4815):882882, Apr. 1953.

205. Malhotra, R., Chan, A., Malhotra, C., and Østbye, T., Prevalence, awareness, treatment and control of hypertension in the elderly population of Singapore. Hypertens. Res. 33(12):1223-1231, 2010.

206. Áurea, R.-S., Pilar, G.-C., José, R. B., and Fernando, R.-A., Relationship between social network and hypertension in older people in Spain. Rev. Española Cardiol. 58(11):1294-1301, 2005.

207. Czaja, S. J., Lee, C. C., Arana, N., Nair, S. N., and Sharit, J., Use of a telehealth system by older adults with hypertension. J. Telemed. Telecare 20(4):184-191, 2014.

208. Chan, M., Estève, D., Fourniols, J.-Y., Escriba, C., and Campo, E., Smart wearable systems: Current status and future challenges. Artif. Intell. Med. 56(3):137-156, 2012.

209. A. T. Agrawal, "Home health monitoring: A review of recent advancements," 2013.

210. Gao, T. et al., The advanced health and disaster aid network: A Light-weight wireless medical system for triage. IEEE Trans. Biomed. Circuits Syst. 1(3):203-216, 2007.

211. Vogeli, C. et al., Multiple chronic conditions: Prevalence, health consequences, and implications for quality, care management, and costs. J. Gen. Intern. Med. 22(3):391-395, 2007.

212. Rocha, A. et al., Innovations in health care services : The CAALYX system. Int. J. Med. Inform.:1-14, 2011.

213. Rekha, R., Mathambigai, T. G., and Vidhyapriya, R., Secure medical data transmission in body area sensor networks using dynamic biometrics and steganography. Bonfring Int. J. Softw. Eng. soft Comput. 2(1):5, 2012.

214. Azeez, D., Ali, M. A. M., Gan, K. B., and Saiboon, I., Comparison of adaptive neuro-fuzzy inference system and artificial neutral networks model to categorize patients in the emergency department. Springerplus 2(1):416, 2013.

215. Brunetti, N. D., Dellegrottaglie, G., Di Giuseppe, G., Antonelli, G., and Di Biase, M., All for one, one for all: Remote telemedicine hub pre-hospital triage for public emergency medical service 1-1-8 in a regional network for primary PCI in Apulia, Italy. Eur. Res. Telemedicine/La Rech. Eur. en Telemed. 3(1):9-15, 2014.

216. Merzougui, R., Adaptation of an intelligent Mobile assistant medical (IMAM) of the heterogeneous data for the telemedicine services: Design and implementation. Wirel. Pers. Commun. 84(4): 3091-3107, 2015.

217. et al Godfrey, B., Emergency Medical Guidelines, Third Edit., no. 3. Sunshine Act of Florida, 2000.

218. Ashour, O. M., and Okudan, G. E., Fuzzy AHP and utility theory based patient sorting in emergency departments. Int. J. Collab. Enterp. 1(3/4):332, 2010.
219. Westergren, H., Ferm, M., and Häggström, P., First evaluation of the paediatric version of the Swedish rapid emergency triage and treatment system shows good reliability. Acta Paediatr. Int. J. Paediatr. 103(3):305-308, 2014.

220. Keeney, R. L., and Raiffa, H., Decisions with multiple objectives: Preferences and value trade-offs. Cambridge university press, 1993.

221. Belton, V., and Stewart, T. J., Multiple criteria decision analysis. Boston, MA: Springer US, 2002.

222. Malczewski, J., GIS and multicriteria decision analysis. John Wiley \& Sons, 1999.

223. Petrovic-Lazarevic, S., and Abraham, A., Hybrid fuzzy-linear programming approach for multi criteria decision making problems. Neural, Parallel Sci. Comput. 11(1 \& 2):53-68, 2003.

224. Khatari, M. et al., Multi-criteria evaluation and benchmarking for active queue management methods: Open issues, challenges and recommended pathway solutions. Int. J. Inf. Technol. Decis. Making., 2019.

225. Zaidan, A. A. et al., Evaluation and selection of open-source EMR software packages based on integrated AHP and TOPSIS. J. Biomed. Inform. 53:390-404, 2015.

226. Zaidan, A. A. et al., Multi-criteria analysis for OS-EMR software selection problem: A comparative study. Decis. Support Syst. 78: 15-27, 2015.

227. Zaidan, B. B. et al., A new digital watermarking evaluation and benchmarking methodology using an external group of evaluators and multi-criteria analysis based on 'large-scale data. Softw. Pract. Exp. 47(10):1365-1392, 2017.

228. Zionts, S., MCDM-If not a Roman numeral, then what? Interfaces (Providence). 9(4):94-101, 1979.

229. Yas, Q. M. et al., Towards on develop a framework for the evaluation and benchmarking of skin detectors based on artificial intelligent models using multi-criteria decision-making techniques. Int. J. Pattern Recognit. Artif. Intell. 31(03):1759002, 2017.

230. Zaidan, B. B. et al., A new approach based on multi-dimensional evaluation and benchmarking for data hiding techniques. International Journal of Information Technology \& Decision Making:1-42, 2017.

231. Baltussen, R., and Niessen, L., Priority setting of health interventions: The need for multi-criteria decision analysis. Cost Eff. Resour. Alloc. 4(1):14, 2006.

232. Thokala, P. et al., Multiple criteria decision analysis for health care decision making - An introduction: Report 1 of the ISPOR MCDA emerging good practices task force. Value Heal. 19(1): $1-13,2016$.

233. Oliveira, M., Fontes, D. B. M. M., and Pereira, T., Multicriteria decision making: A case study in the automobile industry. Ann. Manag. Sci. 3(1):109, 2014.

234. Zaidan, B. B. et al., Software and hardware FPGA-based digital watermarking and steganography approaches: Toward new methodology for evaluation and benchmarking using multi-criteria decision-making. J. Circuits, Syst. Comput., 2017.

235. Jumaah, F. M. et al., Technique for order performance by similarity to ideal solution for solving complex situations in multi-criteria optimization of the tracking channels of GPS baseband telecommunication receivers. Telecommun. Syst.:1-19, 2017.

236. Jadhav, A. and Sonar, R., Analytic hierarchy process (AHP), weighted scoring method (WSM), and hybrid knowledge based system (HKBS) for software selection: A comparative study, in 2009 2nd international conference on emerging trends in engineering and technology, ICETET 2009, 2009, pp. 991-997.

237. Zaidan, A. A. et al., Based multi-agent learning neural network and Bayesian for real-time IoT skin detectors: A new evaluation and benchmarking methodology. Neural Computing and Applications, 2019. 
238. Qader, M. A. et al., A methodology for football players selection problem based on multi-measurements criteria analysis. Meas. J. Int. Meas. Confed. 111:38-50, 2017.

239. Yas, Q. M. et al., Comprehensive insights into evaluation and benchmarking of real-time skin detectors: Review, open issues \&amp; challenges, and recommended solutions. Measurement 114:243-260, Jan. 2018.

240. Alsalem, M. A. et al., Systematic review of an automated multiclass detection and classification system for acute Leukaemia in terms of evaluation and benchmarking, open challenges, issues and methodological aspects. J. Med. Syst. 42(11): 204, Nov. 2018.

241. Albahri, A. S. et al., Real-time fault-tolerant mHealth system: Comprehensive review of healthcare services, opens issues, challenges and methodological aspects, Journal of Medical Systems, vol. 42, no. 8. Springer US, p. 137, Aug-2018.

242. Albahri, O. S. et al., Real-time remote health-monitoring Systems in a Medical Centre: A review of the provision of healthcare services-based body sensor information, open challenges and methodological aspects. J. Med. Syst. 42(9):164, 2018.

243. Alsalem, M. A. et al., Multiclass benchmarking framework for automated acute Leukaemia detection and classification based on BWM and group-VIKOR. J. Med. Syst., 2019.

244. Enaizan, O. et al., Electronic medical record systems: Decision support examination framework for individual, security and privacy concerns using multi-perspective analysis. Heal. Technol., 2018.

245. Adunlin, G., Diaby, V., and Xiao, H., Application of multicriteria decision analysis in health care: A systematic review and bibliometric analysis. Heal. Expect. 18(6):1894-1905, 2015.

246. Diaby, V., Campbell, K., and Goeree, R., Multi-criteria decision analysis (MCDA) in health care: A bibliometric analysis. Oper. Res. Heal. Care 2(1):20-24, 2013.

247. Mühlbacher, A. C., and Kaczynski, A., Making good decisions in healthcare with multi-criteria decision analysis: The use, current research and future development of MCDA. Appl. Health Econ. Health Policy 14(1):29-40, 2016.

248. Tariq, I. et al., MOGSABAT: A metaheuristic hybrid algorithm for solving multi-objective optimisation problems. Neural Comput. Appl., 2018.

249. Almahdi, E. M. et al., Mobile-based patient monitoring systems: A prioritisation framework using multi-criteria decision-making techniques. J. Med. Syst., 2019.

250. Almahdi, E. M. et al., Mobile patient monitoring systems from a benchmarking aspect: Challenges, open issues and recommended solutions. J. Med. Syst., 2019.
251. Zaidan, B. B., and Zaidan, A. A., Comparative study on the evaluation and benchmarking information hiding approaches based multi-measurement analysis using TOPSIS method with different normalisation, separation and context techniques. Measurement 117:277-294, 2018.

252. Zaidan, A. A. et al., "A review on smartphone skin cancer diagnosis apps in evaluation and benchmarking: Coherent taxonomy, open issues and recommendation pathway solution," Health Technol. (Berl)., pp. 1-16, Mar. 2018.

253. Rahmatullah, B. et al., 2017, April. Multi-complex attributes analysis for optimum GPS baseband receiver tracking channels selection. In 2017 4th international conference on control, decision and information technologies (CoDIT) (pp. 1084-1088). IEEE.

254. Salih, M.M., Zaidan, B.B., Zaidan, A.A. and Ahmed, M.A., 2018. Survey on fuzzy TOPSIS state-of-the-art between 2007-2017. Computers \& Operations Research.

255. Jumaah, F. M., Zadain, A. A., Zaidan, B. B., Hamzah, A. K., and Bahbibi, R., Decision-making solution based multi-measurement design parameter for optimization of GPS receiver tracking channels in static and dynamic real-time positioning multipath environment. Measurement 118:83-95, 2018.

256. Opricovic, S., and Tzeng, G.-H., Compromise solution by MCDM methods: A comparative analysis of VIKOR and TOPSIS. Eur. J. Oper. Res. 156(2):445-455, Jul. 2004.

257. M. Mansooreh and J. Pet-Edwards, "Technical briefing: Making multiple-objective decisions," Inst. Electr. ve Electron. Eng. Inc., IEEE Comput. Soc. Press. USA, 1997.

258. Triantaphyllou, E., Multi-criteria decision making methods: A comparative study. Vol. 44. Boston, MA: Springer US, 2000.

259. Aruldoss, M., Lakshmi, T. M., and Venkatesan, V. P., A survey on multi criteria decision making methods and its applications. Am. J. Inf. Syst. 1(1):31-43, 2013.

260. K. P. Yoon and C.-L. Hwang, Multiple attribute decision making: An introduction, vol. 104. Sage publications, 1995.

261. Triantaphyllou, E., Shu, B., Sanchez, S. N., and Ray, T., Multicriteria decision making: An operations research approach. Encycl. Electr. Electron. Eng. 15(1998):175-186, 1998.

262. Abdullateef, B. N. et al., An evaluation and selection problems of OSS-LMS packages. Springerplus 5(1):248, Dec. 2016.

Publisher's Note Springer Nature remains neutral with regard to jurisdictional claims in published maps and institutional affiliations. 